\title{
Computing Bounds of Fractional Metric Dimension of Metal Organic Graphs
}

\author{
Mohsin Raza, ${ }^{1}$ Dalal Awadh Alrowaili, ${ }^{2}$ Muhammad Javaid $\left(\mathbb{D},{ }^{1}\right.$ and Khurram Shabbir ${ }^{3}$ \\ ${ }^{1}$ Department of Mathematics, School of Science, University of Management and Technology, Lahore 54770, Pakistan \\ ${ }^{2}$ Department of Mathematics, College of Science, Jouf University, Sakaka 2014, Saudi Arabia \\ ${ }^{3}$ Department of Mathematics, GC University, Lahore 54000, Pakistan
}

Correspondence should be addressed to Muhammad Javaid; javaidmath@gmail.com

Received 14 January 2021; Revised 10 February 2021; Accepted 27 February 2021; Published 12 March 2021

Academic Editor: Kashif Ali

Copyright ( $\odot 2021$ Mohsin Raza et al. This is an open access article distributed under the Creative Commons Attribution License, which permits unrestricted use, distribution, and reproduction in any medium, provided the original work is properly cited.

Metal organic graphs are hollow structures of metal atoms that are connected by ligands, where metal atoms are represented by the vertices and ligands are referred as edges. A vertex $x$ resolves the vertices $u$ and $v$ of a graph $G$ if $d(u, x) \neq d(v, x)$. For a pair $(u, v)$ of vertices of $G, R(u, v)=\{x \in V(G): d(x, u) \neq d(x, v)\}$ is called its resolving neighbourhood set. For each pair of vertices $u$ and $v$ in $V(G)$, if $f(R(u, v)) \geq 1$, then $f$ from $V(G)$ to the interval [0,1] is called resolving function. Moreover, for two functions $f$ and $g, f$ is called minimal if $f \leq g$ and $f(v) \neq g(v)$ for at least one $v \in V(G)$. The fractional metric dimension (FMD) of $G$ is denoted by $\operatorname{dim}_{f}(G)$ and defined as $\operatorname{dim}_{f}(G)=\min \{|g|: g$ is a minimal resolving function of $G\}$, where $|g|=\sum_{v \in V(G)} g(v)$. If we take a pair of vertices $(u, v)$ of $G$ as an edge $e=u v$ of $G$, then it becomes local fractional metric dimension (LFMD) $\left(\operatorname{dim}_{l f}(G)\right)$. In this paper, local fractional and fractional metric dimensions of MOG $(n)$ are computed for $n \cong 1(\bmod 2)$ in the terms of upper bounds. Moreover, it is obtained that metal organic is one of the graphs that has the same local and fractional metric dimension.

\section{Introduction}

For a connected graph $G$, a vertex $x \in V(G)$ is said to resolve a pair $(u, v)$ of vertices of $G$ if $d(x, u) \neq d(x, v)$. A set $S \subseteq V(G)$ is called a resolving set of $G$ if each pair of vertices of $G$ is resolved by some vertex in S. The metric dimension of $G$ is denoted by $\operatorname{dim}(G)$ and is defined as

$$
\operatorname{dim}(G)=\min \{|S|: S \text { is a resolving set of } G\} .
$$

For a pair $(u, v)$ of vertices of $G$, the resolving neighborhood $R(u, v)$ is defined as $R(u, v)=\{w \in V(G): d(w, u) \neq$ $d(w, v)\}$. A resolving function is a real-valued function $g: V(G) \longrightarrow[0,1]$ such that $g(R(u, v)) \geq 1$ for each distinct pair of vertices of $G$, where $g(R(u, v))=\sum_{x \in R(u, v)} g(x)$. A resolving function $g$ is called minimal if any function $f: V(G) \longrightarrow[0,1]$ such that $f \leq g$ and $f(v) \neq g(v)$ for at least one $v \in V$ is not a resolving function of $G$. The fractional metric dimension (FMD) of $G$ is denoted by $\operatorname{dim}_{f}(G)$ and defined as
$\operatorname{dim}_{f}(G)=\min \{|g|: g$ is a minimal resolving function of $G\}$,

where $|g|=\leq \sum_{v \in V(G)} g(v)$. Now, if we take a pair of vertices $(u, v)$ of $G$ as an edge $e=u v$ of $G$, then the aforesaid defined resolving neighborhood $R(u, v)$, minimal resolving function $g$, and FMD $\operatorname{dim}_{f}(G)$ become local resolving neighborhood $(\mathrm{LR}(u v))$, local minimal resolving function, and local fractional metric dimension $\left(\operatorname{dim}_{l f}(G)\right)$, respectively.

First of all, Harary and Melter [1] defined the concept of metric dimension to study the substructures of chemical compounds having similar properties which are used in pharmaceutical industries for the drug discoveries. Later on, Chartrand et al. [2] and Currie \& Oellermann [3, 4] improved the solution of IPP with the help of the procedure of metric dimension. Moreover, it is used in navigation system, image processing, and robotic problems [5]. For various results of metric dimension on different graphs, refer to [6-9]. 
Fehr et al. [10] introduced the concept of fractional metric dimension (FMD), and they obtained the optimal solution of a certain linear programming relaxation problem with the help of FMD. Arumugam and Mathew [11] present various properties of FMD. The FMD of metal organic framework (MOF) is computed in [12], where MOF is obtained from the cycle of odd order. Moreover, different classes of graphs such as product-based graphs and Hamming, Johnson, and permutation graphs are studied with the help of FMD [13-17]. Liu et al. [18] computed the FMD of generalized Jahangir graph. Recently, Aisyah et al. defined the concept of local fractional metric dimension (LFMD) and computed it for the corona product of graphs [19]. Liu et al. [20] computed the LFMD of rotationally symmetric networks. Javaid et al. [21] calculated the sharp bounds of LFMD of connected networks.

Metal organic graph (MOG) consists of metal atoms, where atoms are linked with thes help of organic ligands which act like a linker. Therefore, MOG has led to a new world of remarkable applications and it has a large surface area that allows these chemicals compounds to absorb huge quantity of several gases such as carbon dioxide hydrogen and methane acting as a gas storage chemical compound. It is also utilized for environmental protection and cleaning energy with the help of capturing carbon dioxide. Being small density, high surface structure flexibility, and tuneable pore functionality, metal organic frameworks also play an important role in liquid-phase separation that is industrial step with critical roles in petrochemical, chemical, nuclear, and pharmaceutical industries. These frame works are also used in heterogeneous catalyst, drugs delivery, and sensing conductivity [22-25].

In this paper, upper bounds for LFMD and FMD of the metal organic graphs are calculated, where MOGs are obtained with the help of the cycles of even order. Moreover, the unboundedness of the obtained results is also discussed. Rest of the paper is organized as follows: Section 1 includes the introduction. Construction of MOG is discussed in Section 2. LFMD of metal organic graphs is added in Section 3. FMD of MOG is calculated in Section 4. Conclusion is presented in Section 5.

\section{Construction of Metal Organic Graphs}

In this section, we describe the construction of metal organic graphs. Let $\operatorname{MOG}(n)$ for $n \geq 3$ be a metal organic graph with vertex set $V(\operatorname{MOG}(n))=\left\{u_{i}: 1 \leq i \leq n\right\} \cup\left\{v_{j}: 1 \leq j \leq 2 n\right\}$ and edge set $E(\operatorname{MOG}(n))=\left\{u_{i} u_{i+1}: 1 \leq i \leq n-\quad 1\right\} \cup$ $\left\{v_{j} v_{j+1}: 1 \leq j \leq 2 n-1\right\} \cup\left\{u_{n} u_{1}, v_{2 n} v_{1}\right\} \cup\left\{u_{i} v_{j}, u_{i} v_{j+1}: 1 \leq\right.$ $i \leq n, 1 \leq j \leq 2 n\}$, where $|V(\operatorname{MOG}(n))|=E|(\operatorname{MOG}(n))|=3 n$. Figure 1 shows $\operatorname{MOG}(n)$ for $n \in\{5,7,9\}$.

\section{LFMD of Metal Organic Graphs}

In this section, local resolving neighbourhood sets of metal organic graphs are discussed in Lemmas 1 and 2 and local fractional metric dimension is calculated in Theorem 1.
Lemma 1. Let $M O G(n)$ for $n \equiv 1(\bmod 2)$ and $n \geq 5$ be metal organic graph, then $\left|L R\left(e_{t}\right)\right|=\left|L R\left(e_{t}=v_{i} v_{j}\right)\right|=8$. For $1 \leq k \leq n, \quad j=i+1, \quad i \in[2 k-1], \quad 1 \leq t \leq n$. Moreover, $\cup L R\left(e_{t}\right)=\left\{v_{m}: 1 \leq m \leq 2 n\right\}$ and $\left|\cup L R\left(e_{t}\right)\right|=\alpha=2 n$.

Proof. The local resolving neighborhood of metal organic graphs, for $1 \leq k \leq n, \quad j=i+1, \quad i \in[2 k-1], \quad 1 \leq t \leq n$. $\operatorname{LR}\left(v_{i} v_{j}\right)=\left\{v_{l}: 2 k-1 \leq l \leq k-3,2 k-4 \leq l \leq 2 k-2\right\} \quad$ with $\left|\operatorname{LR}\left(e_{t}\right)\right|=8$ and $\cup_{t=1}^{n} \operatorname{LR}\left(e_{t}\right)=\left\{v_{s}: 1 \leq s \leq 2 n\right\}$, and we have $\left|\cup_{t=1}^{n} \operatorname{LR}\left(e_{t}\right)\right|=2 n$.

Lemma 2. Let $\operatorname{MOG}(n)$ for $n \equiv 1(\bmod 2)$ and $n \geq 9$ be a metal organic graph with $1 \leq t \leq n$. Then, the following holds:

(a) For $1 \leq k \leq n, j=i+1, i \in[2 k],\left|L R\left(e_{t}\right)\right|<\left|L R\left(v_{i} v_{j}\right)\right|$ and $\mid L R\left(v_{i} v_{j}\right) \cap\left(\cup_{t=1}^{n} \operatorname{LR}\left(e_{t}\right)|\geq| L R\left(e_{t}\right) \mid\right.$.

(b) For $1 \leq i \leq n-1, j=i+1,\left|L R\left(e_{t}\right)\right|<\left|L R\left(u_{i} u_{j}\right)\right|$ and $\mid \operatorname{LR}\left(u_{i} u_{j}\right) \cap\left(\cup_{t=1}^{n} \operatorname{LR}\left(e_{t}\right)|\geq| \operatorname{LR}\left(e_{t}\right) \mid\right.$.

(c) For $1 \leq i \leq n, j=2 i-1,2 i,\left|L R\left(e_{t}\right)\right|<\left|L R\left(u_{i} u_{j}\right)\right|$ and $\mid \operatorname{LR}\left(u_{i} u_{j}\right) \cap\left(\cup_{t=1}^{n} \operatorname{LR}\left(e_{t}\right)|\geq| \operatorname{LR}\left(e_{t}\right) \mid\right.$.

(d) For $\quad 1 \leq i \leq n, \quad j=2 i,\left|\operatorname{LR}\left(e_{t}\right)\right|<\left|L R\left(u_{i} u_{j}\right)\right| \quad$ and $\mid \operatorname{LR}\left(u_{i} u_{j}\right) \cap\left(\cup_{t=1}^{n} \operatorname{LR}\left(e_{t}\right)|\geq| \operatorname{LR}\left(e_{t}\right) \mid\right.$.

Proof. (a) The local resolving neighborhood for $1 \leq k \leq n$, $j=i+1, i \in[2 k], 1 \leq t \leq n$,

$\operatorname{LR}\left(v_{i} v_{j}\right)= \begin{cases}u_{p}: & 1 \leq p \leq n, \text { where } p \neq \frac{n+1}{2}+k=m, \\ v_{q}: & 1 \leq q \leq 2 n, \text { where } q \neq 2 m, 2 m-1,\end{cases}$

with $\left|\operatorname{LR}\left(v_{i} v_{j}\right)\right|=3 n-3>8=\left|\operatorname{LR}\left(e_{t}\right)\right|, \quad \operatorname{LR}\left(v_{i} v_{j}\right) \cap$ $\left(\cup_{t=1}^{n} \operatorname{LR} e_{t}\right)=\left\{v_{q}: 1 \leq q \leq 2 n, q \neq 2 m, 2 m-1\right\}$.

Therefore,

$\left|\operatorname{LR}\left(v_{i} v_{j}\right) \cap\left(\cup_{t=1}^{n} \mathrm{LR}_{t}\right)\right|=2 n-2>\left|\operatorname{LR}\left(e_{t}\right)\right|$.

(b) The local resolving neighborhood for $1 \leq i \leq n-1$, $j=i+1,1 \leq t \leq n$,

$\operatorname{LR}\left(u_{i} u_{j}\right)= \begin{cases}u_{p}: & 1 \leq p \leq n, \text { where } p \neq \frac{n+i+j}{2}+k=m, \\ v_{q}: & 1 \leq q \leq 2 n, \text { where } q \neq 2 m, 2 m-1,\end{cases}$

with $\left|\operatorname{LR}\left(u_{i} u_{j}\right)\right|=3 n-3>8=\left|\operatorname{LR}\left(e_{t}\right)\right| \quad$ and $\operatorname{LR}\left(u_{i} u_{j}\right) \cap \quad\left(\cup_{t=1}^{n} \operatorname{LRe} e_{t}\right)=\left\{v_{q}: 1 \leq q \leq 2 n, \quad q \neq 2 m\right.$, $2 m-1\}$. Therefore, we have $\mid \operatorname{LR}\left(u_{i} u_{j}\right) \cap$ $\left(\cup_{t=1}^{n} \operatorname{LR} e_{t}\right)|=2 n-2>| \operatorname{LR}\left(e_{t}\right) \mid$.

(c) The local resolving neighborhood for $1 \leq i \leq n$, $j=2 i-1,1 \leq t \leq n$,

$\operatorname{LR}\left(u_{i} v_{j}\right)= \begin{cases}u_{p}: & 1 \leq p \leq n ; \\ v_{q}: & 1 \leq q \leq 2 n \text { where } q \neq j+1,2,-2,-3,\end{cases}$ 


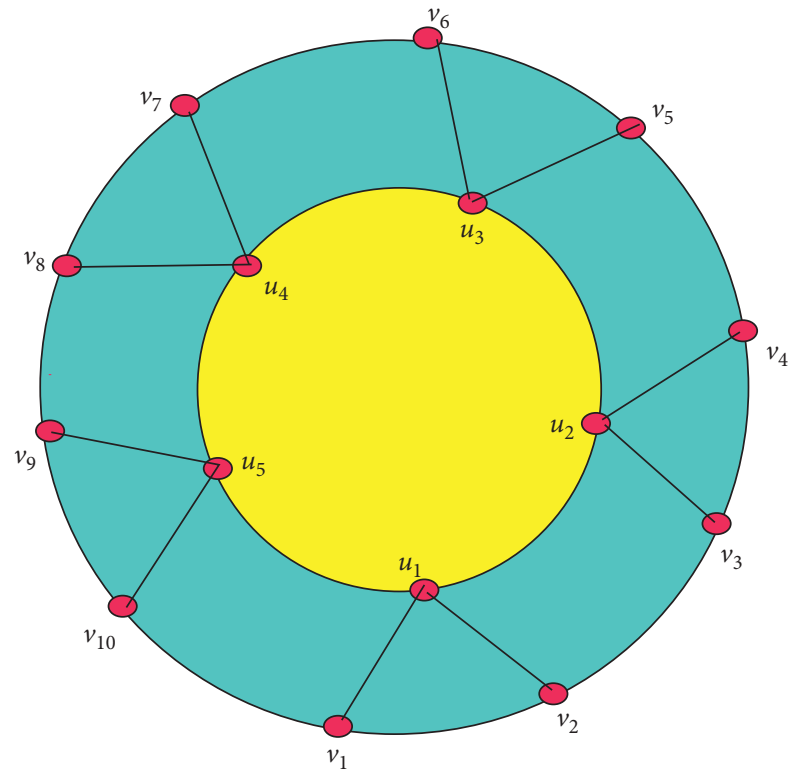

(a)

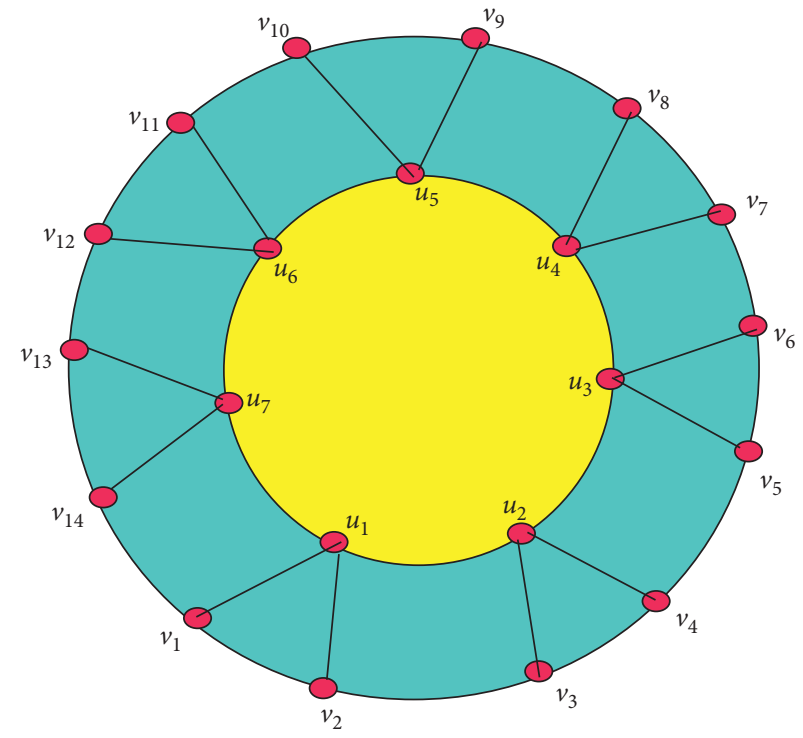

(b)

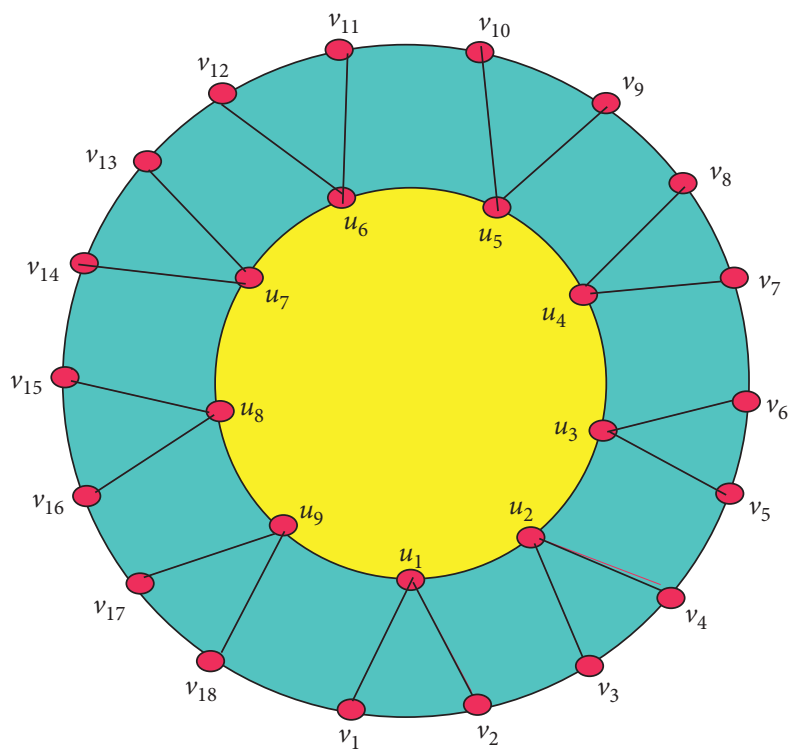

(c)

Figure 1: $\operatorname{MOG}(n)$ for $n=5$ (a), $n=7$ (b), and $n=9$ (c).

with $\quad\left|\operatorname{LR}\left(u_{i} u_{j}\right)\right|=3 n-4>8=\left|\operatorname{LR}\left(e_{t}\right)\right| \quad$ and $\operatorname{LR}\left(u_{i} u_{j}\right) \cap\left(\cup_{t=1}^{n} \operatorname{LR} e_{t}\right)=\left\{v_{q}: q \neq j+1,2,-2,-3\right\}$. Therefore, we have $\mid \operatorname{LR}\left(u_{i} u_{j}\right)$ $\cap\left(\cup_{t=1}^{n} \operatorname{LR} e_{t}\right)|=2 n-4>| \operatorname{LR}\left(e_{t}\right) \mid$

(d) The local resolving neighborhood for $1 \leq i \leq n$, $j=2 i-1,1 \leq t \leq n$,

$\operatorname{LR}\left(u_{i} v_{j}\right)= \begin{cases}u_{p}: & 1 \leq p \leq n ; \\ v_{q}: & 1 \leq q \leq 2 n, \text { where } q \neq j+2,3,-1,-2,\end{cases}$

with $\left|\operatorname{LR}\left(u_{i} u_{j}\right)\right|=3 n-4>8=\left|\operatorname{LR}\left(e_{t}\right)\right| \quad$ and $\operatorname{LR}\left(u_{i} u_{j}\right) \cap\left(\cup_{t=1}^{n} \operatorname{LR} e_{t}\right)=\left\{v_{q}: q \neq j+2,3,-1,-2\right\}$.
Therefore, we have $\left|\operatorname{LR}\left(u_{i} u_{j}\right) \cap \quad\left(\cup_{t=1}^{n} \mathrm{LR} e_{t}\right)\right|=$ $2 n-4>\left|\operatorname{LR}\left(e_{t}\right)\right|$.

Theorem 1. Let $M O G(n)$ for $n \equiv 1(\bmod 2)$ and $n \geq 5$ be the metal organic graphs, then $\operatorname{dim}_{l f}(M O G(n)) \leq n / 4$.

Proof. In view of Lemmas 1 and 2 for for $1 \leq k \leq n, j=i+1$, $i \in[2 k-1], 1 \leq t \leq n,\left|\operatorname{LR}\left(e_{t}\right)\right|=\left|\operatorname{LR}\left(v_{i} v_{j}\right)\right|=8$ and $|X|=$ $\left|\cup_{t=1}^{n} \operatorname{LR}\left(e_{t}\right)\right|=2 n$.

We have $|R(x y)| \leq\left|R\left(e_{t}\right)\right|$ for all $x y \in E(\operatorname{MOG}(n))$. Moreover, the local resolving neighbourhood of minimum cardinality is not disjoint. Therefore, local fractional metric of $\operatorname{MOG}(n)$ is given as follows: 


$$
\operatorname{dim}_{l f}(\operatorname{MOG}(n)) \leq \sum_{t=1}^{|X|} \frac{1}{\left|\operatorname{LR}\left(e_{t}\right)\right|}
$$

For $|X|=2 n$ and $\left|\operatorname{LR}\left(e_{t}\right)\right|=8$, we have

$$
\operatorname{dim}_{l f}(\operatorname{MOG}(n)) \leq \sum_{t=1}^{2 n} \frac{1}{8} .
$$

Hence, $\operatorname{dim}_{l f}(\operatorname{MOG}(n)) \leq n / 4$.

\section{FMD of Metal Organic Graphs}

In this section, the resolving neighbourhood sets of metal organic graphs are calculated in Lemmas 3-8. Bounds of FMD are computed in Theorems 2 and 3.

Lemma 3. Let $M O G(n)$ for $n \equiv 1(\bmod 2)$ and $n \geq 9$ be metal organic graph, then $\left|R\left(e_{t}\right)\right|=\left|R\left(e_{t}=v_{i}, v_{j}\right)\right|=8$. For $1 \leq k \leq n, \quad j=i+1, \quad i \in[2 k-1], \quad 1 \leq t \leq n$. Moreover, $\cup_{t=1}^{n} R\left(e_{t}\right)=\left\{v_{m}: 1 \leq m \leq 2 n\right\}$ and $\left|\cup_{t=1}^{n} R\left(e_{t}\right)\right|=\alpha=2 n$.

Proof. The resolving neighborhood sets of metal organic graph for $1 \leq k \leq n, \quad j=i+1, \quad i \in[2 k-1], \quad 1 \leq t \leq n$, $R\left(v_{i}, v_{j}\right)=\left\{v_{l}: 2 k-1 \leq l \leq k-3,2 k-4 \leq l \leq 2 k-2\right\} \quad$ with $\left|R\left(e_{t}\right)\right|=8$ and $\cup_{t=1}^{n} R\left(e_{t}\right)=\left\{v_{s}: 1 \leq s \leq 2 n\right\}$, and we have $\left|\cup_{t=1}^{n} R\left(e_{t}\right)\right|=2 n$.

Lemma 4. Let $\operatorname{MOG}(n)$ for $n \equiv 1(\bmod 2)$ and $n \geq 9$ be metal organic graphs, then for $1 \leq k \leq n, i \in[2 k-1], 1 \leq t \leq n$, $\left|R\left(e_{t}\right)\right|<\left|R\left(v_{i}, v_{j}\right)\right|$ and $\mid R\left(v_{i}, v_{j}\right) \cap\left(\cup_{t=1}^{n} R\left(e_{t}\right)|\geq| R\left(e_{t}\right) \mid\right.$ :
(a) $j \in\{i+1\}$.
(b) $j \in\{i+2, i+6\}$.
(c) $j \in\{i+3, i+7\}$.
(d) $j \in\{i+4, i+8\}$.
(e) $j \in\{i+5\}$.

Proof. (a) The resolving neighborhood for $1 \leq k \leq n$, $i \in[2 k-1] j \in i+1,1 \leq t \leq n$,

$R\left(v_{i}, v_{j}\right)= \begin{cases}u_{p}: & 1 \leq p \leq n, \text { where } p \neq \frac{n+1}{2}+k=m, \\ v_{q}: & 1 \leq q \leq 2 n, \text { where } q \neq 2 m, 2 m-1,\end{cases}$

with $\left|R\left(v_{i}, v_{j}\right)\right|=3 n-3>8=\left|R\left(e_{t}\right)\right| \quad$ and $R\left(v_{i}, v_{j}\right) \cap\left(\cup_{t=1}^{n} \operatorname{Re}_{t}\right)=\left\{v_{q}: 1 \leq q \leq 2 n: \quad q \neq 2 m\right.$, $2 m-1\}$. Therefore, we have $\left|R\left(v_{i}, v_{j}\right) \cap\left(\cup_{t=1}^{n} R e_{t}\right)\right|=2 n-2>\left|R\left(e_{t}\right)\right|$.

(b) The resolving neighborhood for $1 \leq k \leq n$, $i \in[2 k-1] j \in i+2, i+6,1 \leq t \leq n$.

When $j \in\{i+2\}$,
$R\left(v_{i}, v_{j}\right)= \begin{cases}u_{p}: & 1 \leq p \leq n, \text { where } p \neq \frac{n+2 i+1}{2}=m, \\ v_{q}: & 1 \leq q \leq 2 n, \text { where } q \neq 2 m, 2 m-1, i+1 .\end{cases}$

When $j \in\{i+6\}$,

$R\left(v_{i}, v_{j}\right)= \begin{cases}u_{p}: & 1 \leq p \leq n, \text { where } p \neq \frac{n+2 i+3}{2}=m, \\ v_{q}: & 1 \leq q \leq 2 n, \text { where } q \neq 2 m, 2 m-1, i+3,\end{cases}$

with $\quad\left|R\left(v_{i}, v_{j}\right)\right|=3 n-4>8=\left|R\left(e_{t}\right)\right|$, $R\left(v_{i}, v_{j}\right) \cap\left(\cup_{t=1}^{n} R e_{t}\right)=\left\{v_{q}: 1 \leq q \leq 2 n: q \neq 2 m\right.$, $2 m-1, i+1\}$, and $j \in i+2 .\left|R\left(v_{i}, v_{j}\right) \cap\left(\cup_{t=1}^{n} R e_{t}\right)\right|=$ $\left\{v_{q}: 1 \leq q \leq 2 n: q \neq 2 m, 2 m-1, i+3\right\}$ and $j \in i+6$. Therefore, we have $\left|R\left(v_{i}, v_{j}\right) \cap\left(\cup_{t=1}^{n} R e_{t}\right)\right|$ $=2 n-3>\left|R\left(e_{t}\right)\right|$.

(c) The resolving neighborhood for $1 \leq k \leq n$, $i \in[2 k-1] j \in i+3, i+7,1 \leq t \leq n$.

When $j \in\{i+3\}$,

$R\left(v_{i}, v_{j}\right)= \begin{cases}u_{p}: & 1 \leq p \leq n, \text { where } p \neq \frac{n+2 i+1}{2}=m, \\ v_{q}: & 1 \leq q \leq 2 n, \text { where } q \neq 2 m, 2 m-1 .\end{cases}$

When $j \in\{i+7\}$,

$R\left(v_{i}, v_{j}\right)= \begin{cases}u_{p}: & 1 \leq p \leq n, \text { where } p \neq \frac{n+2 i+3}{2}=m, \\ v_{q}: & 1 \leq q \leq 2 n, \text { where } q \neq 2 m, 2 m-1,\end{cases}$

with $\left|R\left(v_{i}, v_{j}\right)\right|=3 n-3>8=\left|R\left(e_{t}\right)\right|, \quad R\left(v_{i}, v_{j}\right) \cap$ $\left(\cup_{t=1}^{n} R e_{t}\right)=\left\{v_{q}: 1 \leq q \leq 2 n: \quad q \neq 2 m, 2 m-1\right\}, \quad$ and $j \in i+3 . \quad R\left(v_{i}, v_{j}\right) \cap\left(\cup_{t=1}^{n} R e_{t}\right)=\left\{v_{q}: 1 \leq q \leq 2 n:\right.$ $q \neq 2 m, 2 m-1\}$ and $j \in i+7$. Therefore, we have $\left|R\left(v_{i}, v_{j}\right) \cap\left(\cup_{t=1}^{n} R e_{t}\right)\right|=2 n-2>\left|R\left(e_{t}\right)\right|$.

(d) The resolving neighborhood for $1 \leq k \leq n$, $i \in[2 k-1] j \in i+4, i+8,1 \leq t \leq n$.

When $j \in\{i+4\}$,

$R\left(v_{i}, v_{j}\right)= \begin{cases}u_{p}: & 1 \leq p \leq n, \text { where } p \neq \frac{j-1}{2}, \\ v_{q}: & 1 \leq q \leq 2 n, \text { where } q \neq \frac{i+j}{2} .\end{cases}$

When $j \in\{i+8\}$, 


$$
R\left(v_{i}, v_{j}\right)= \begin{cases}u_{p}: & 1 \leq p \leq n, \text { where } p \neq \frac{j-3}{2}, \\ v_{q}: & 1 \leq q \leq 2 n, \text { where } q \neq \frac{i+j}{2},\end{cases}
$$

with $\left|R\left(v_{i}, v_{j}\right)\right|=3 n-2>8=\left|R\left(e_{t}\right)\right|$ and $R\left(v_{i}, v_{j}\right) \cap$ $\left(\cup_{t=1}^{n} R e_{t}\right)=\left\{v_{q}: 1 \leq q \leq 2 n: q \neq i+j / 2\right\}$. Therefore, we have $\left|R\left(v_{i}, v_{j}\right) \cap\left(\cup_{t=1}^{n} R e_{t}\right)\right|=2 n-1>\left|R\left(e_{t}\right)\right|$.

(e) The resolving neighborhood for $1 \leq k \leq n$, $i \in[2 k-1] j \in i+5,1 \leq t \leq n$.

$R\left(v_{i}, v_{j}\right)= \begin{cases}u_{p}: & 1 \leq p \leq n, \text { where } p \neq \frac{j-2}{2}, \\ v_{q}: & 1 \leq q \leq 2 n,\end{cases}$

with $\left|R\left(v_{i}, v_{j}\right)\right|=3 n-1>8=\left|R\left(e_{t}\right)\right|$ and $R\left(v_{i}, v_{j}\right) \cap$ $\left(\cup_{t=1}^{n} R e_{t}\right)=\left\{v_{q}: 1 \leq q \leq 2 n\right\}$. Therefore, we have $\left|R\left(v_{i}, v_{j}\right) \cap\left(\cup_{t=1}^{n} R e_{t}\right)\right|=2 n>\left|R\left(e_{t}\right)\right|$.

Lemma 5. Let $M O G(n)$ for $n \equiv 1(\bmod 2)$ and $n \geq 9$ be metal organic graph. Then, for $1 \leq k \leq n, \quad i \in[2 k], \quad 1 \leq t \leq n$, $\left|R\left(e_{t}\right)\right|<\left|R\left(v_{i}, v_{j}\right)\right|$ and $\left|R\left(v_{i}, v_{j}\right) \cap\left(\cup_{t=1}^{n} R e_{t}\right)\right| \geq\left|R\left(e_{t}\right)\right|:$
(a) $j \in\{i+2, i+6\}$.
(b) $j \in\{i+3, i+7\}$.
(c) $j \in\{i+4, i+8\}$.
(d) $j \in\{i+5\}$.

Proof. (a) The resolving neighborhood for $1 \leq k \leq n$, $i \in[2 k] j \in i+2, i+6,1 \leq t \leq n$.

When $j \in\{i+2\}$,

$$
R\left(v_{i}, v_{j}\right)= \begin{cases}u_{p}: & 1 \leq p \leq n, \text { where } p \neq \frac{n+i+1}{2}=m, \\ v_{q}: & 1 \leq q \leq 2 n, \text { where } q \neq 2 m, 2 m-1, i+1 .\end{cases}
$$

When $j \in\{i+6\}$,

$$
R\left(v_{i}, v_{j}\right)= \begin{cases}u_{p}: & 1 \leq p \leq n, \text { where } p \neq \frac{n+i+3}{2}=m, \\ v_{q}: & 1 \leq q \leq 2 n, \text { where } q \neq 2 m, 2 m-1, i+3,\end{cases}
$$

with $\left|R\left(v_{i}, v_{j}\right)\right|=3 n-4>8=\left|R\left(e_{t}\right)\right|, \quad R\left(v_{i}, v_{j}\right) \cap$ $\left(\cup_{t=1}^{n} R e_{t}\right)=\left\{v_{q}: 1 \leq q \leq 2 n: q \neq 2 m, 2 m-1, i+1\right\}$, and $j \in i+2 . \quad R\left(v_{i}, v_{j}\right) \cap \quad\left(\cup_{t=1}^{n} R e_{t}\right)=\left\{v_{q}: 1 \leq\right.$ $q \leq 2 n: \quad q \neq 2 m, 2 m-1, i+3\}$ and $j \in i+6$. Therefore, we have $\left|R\left(v_{i}, v_{j}\right) \cap\left(\cup_{t=1}^{n} R e_{t}\right)\right|=2 n-3>\left|R\left(e_{t}\right)\right|$.

(b) The resolving neighborhood for $1 \leq k \leq n$, $i \in[2 k] j \in i+3, i+7,1 \leq t \leq n$.

$$
R\left(v_{i}, v_{j}\right)= \begin{cases}u_{p}: & 1 \leq p \leq n, \text { where } p \neq \frac{j+i+1}{4}, \\ v_{q}: & 1 \leq q \leq 2 n,\end{cases}
$$

with $\quad\left|R\left(v_{i}, v_{j}\right)\right|=3 n-1>8=\left|R\left(e_{t}\right)\right| \quad$ and $R\left(v_{i}, v_{j}\right) \cap\left(\cup_{t=1}^{n} R e_{t}\right)=\left\{v_{q}: 1 \leq q \leq 2 n\right\}$. Therefore, we have $\left|R\left(v_{i}, v_{j}\right) \cap\left(\cup_{t=1}^{n} R e_{t}\right)\right|=2 n>\left|R\left(e_{t}\right)\right|$.

(c) The resolving neighborhood for $1 \leq k \leq n$, $i \in[2 k] j \in i+4, i+8,1 \leq t \leq n$,

$R\left(v_{i}, v_{j}\right)= \begin{cases}u_{p}: & 1 \leq p \leq n, \text { where } p \neq \frac{i+j}{4}, \\ v_{q}: & 1 \leq q \leq 2 n: q \neq \frac{i+j}{2},\end{cases}$

with $\left|R\left(v_{i}, v_{j}\right)\right|=3 n-2>8=\left|R\left(e_{t}\right)\right|$ and $R\left(v_{i}, v_{j}\right) \cap$ $\left(\cup_{t=1}^{n} R e_{t}\right)=\left\{v_{q}: 1 \leq q \leq 2 n: q \neq i+j / 2\right\}$. Therefore, we have $\left|R\left(v_{i}, v_{j}\right) \cap\left(\cup_{t=1}^{n} R e_{t}\right)\right|=2 n-1>\left|R\left(e_{t}\right)\right|$.

(d) The resolving neighborhood for $1 \leq k \leq n$, $i \in[2 k] j \in\{i+5\}, 1 \leq t \leq n$.

$$
R\left(v_{i}, v_{j}\right)= \begin{cases}u_{p}: & 1 \leq p \leq n, \text { where } p \neq \frac{n+i+3}{2}=m, \\ v_{q}: & 1 \leq q \leq 2 n, \text { where } q \neq 2 m, 2 m-1,\end{cases}
$$

with $\left|R\left(v_{i}, v_{j}\right)\right|=3 n-3>8=\left|R\left(e_{t}\right)\right|$ and $R\left(v_{i}, v_{j}\right) \cap$ $\left(\cup_{t=1}^{n} R e_{t}\right)=\left\{v_{q}: 1 \leq q \leq 2 n: q \neq 2 m, 2 m-1\right\}$.

Therefore we

have $\left|R\left(v_{i}, v_{j}\right) \cap\left(\cup_{t=1}^{n} R e_{t}\right)\right|=2 n-2>\left|R\left(e_{t}\right)\right|$.

Lemma 6. Let $M O G(n)$ for $n \equiv 1(\bmod 2)$ and $n \geq 9$ be metal organic graph. Then, the following holds.

(a) For $\quad 1 \leq k \leq n, \quad j=i+1, \quad i \in[2 k], \quad 1 \leq t \leq n$, $\left|R\left(e_{t}\right)\right|<\left|R\left(v_{i} v_{j}\right)\right|$ and $\left|R\left(v_{i}, v_{j}\right) \cap \quad\left(\cup_{t=1}^{n} R e_{t}\right)\right|=$ $\geq\left|R\left(e_{t}\right)\right|$.

Proof. The resolving neighborhood for $1 \leq i \leq n$, $j \in\{9+i+k: 1 \leq k \leq 2 n-18\}$.

When $k=4 t-3,4 t-2$, for $1 \leq t \leq n-9 / 2$,

$$
R\left(v_{i}, v_{j}\right)= \begin{cases}u_{p}: & 1 \leq p \leq n, \text { where } p \neq \frac{n+i+6}{2}=m, \\ v_{q}: & 1 \leq q \leq 2 n, \text { where } q \neq 2 m, 2 m-1 .\end{cases}
$$

When $k=4 t-1,4 t$, for $1 \leq t \leq n-9 / 2$,

$$
R\left(v_{i}, v_{j}\right)= \begin{cases}u_{p}: & 1 \leq p \leq n, \text { where } p \neq \frac{i+7}{2}=m, \\ v_{q}: & 1 \leq q \leq 2 n, \text { where } q \neq 2 m, 2 m-1,\end{cases}
$$


with $\left|R\left(v_{i}, v_{j}\right)\right|=3 n-3>8=\left|R\left(e_{t}\right)\right|$ and $R\left(v_{i}, v_{j}\right) \cap$ $\left(\cup_{t=1}^{n} R e_{t}\right)=\left\{v_{q}: 1 \leq q \leq 2 n: q \neq 2 m, 2 m-1\right\}$. Therefore, we have $\left|R\left(v_{i}, v_{j}\right) \cap\left(\cup_{t=1}^{n} R e_{t}\right)\right|=2 n-2>\left|R\left(e_{t}\right)\right|$.

\section{Corollary 1.}

(i) For $1 \leq k \leq n, \quad i \in[2 k-1],\left|R\left(v_{i}, v_{j}\right)\right|=\left|R\left(v_{i}, v_{m}\right)\right|$, where $j \in\{i+s: 2 \leq s \leq 8\}$ and $m \in\{i-s: 2 \leq s \leq 8\}$.

(ii) For $1 \leq k \leq n, i \in[2 k]\left|R\left(v_{i}, v_{j}\right)\right|=\left|R\left(v_{i}, v_{j}\right)\right|$, where $j \in\{i+s, i-s: 2 \leq s \leq 7\}$.

Lemma 7. Let $\operatorname{MOG}(n)$ for $n \equiv 1(\bmod 2)$ and $n \geq 9$ be metal organic graph. Then, for $1 \leq i \leq n, 1 \leq k \leq n-1, j=i+k$, $1 \leq t \leq n, \quad\left|R\left(e_{t}\right)\right|<\left|R\left(u_{i} u_{j}\right)\right| \quad$ and $\quad \mid R\left(u_{i}, u_{j}\right) \cap$ $\left(\cup_{t=1}^{n} R e_{t}\right)|=\geq| R\left(e_{t}\right) \mid$.

Proof. The resolving neighborhood for $1 \leq i \leq n$, $1 \leq k \leq n-1, j=i+k$.

When $k \cong 1(\bmod 2)$,

$R\left(u_{i}, u_{j}\right)= \begin{cases}u_{p}: & 1 \leq p \leq n, \text { where } p \neq \frac{n+i+j}{2}=m, \\ v_{q}: & 1 \leq q \leq 2 n, \text { where } q \neq 2 m, 2 m-1 .\end{cases}$

When $k \cong 0(\bmod 2)$,

$R\left(u_{i}, u_{j}\right)= \begin{cases}u_{p}: & 1 \leq p \leq n, \text { where } p \neq \frac{i+j}{2}=m, \\ v_{q}: & 1 \leq q \leq 2 n, \text { where } q \neq 2 m, 2 m-1,\end{cases}$ with $\left|R\left(u_{i}, u_{j}\right)\right|=3 n-3>8=\left|R\left(e_{t}\right)\right|$ and $R\left(u_{i}, u_{j}\right) \cap$ $\left(\cup_{t=1}^{n} R e_{t}\right)=\left\{v_{q}: 1 \leq q \leq 2 n, q \neq 2 m, 2 m-1\right\}$. Therefore, we have $\left|R\left(u_{i}, u_{j}\right) \cap\left(\cup_{t=1}^{n} R e_{t}\right)\right|=2 n-2>\left|R\left(e_{t}\right)\right|$.

Lemma 8. Let $\operatorname{MOG}(n)$ for $n \equiv 1(\bmod 2)$ and $n \geq 9$ be metal organic graph. Then, for $1 \leq i \leq n,\left|R\left(e_{t}\right)\right|<\left|R\left(u_{i}, v_{j}\right)\right|$ and $\left|R\left(u_{i}, u_{j}\right) \cap\left(\cup_{t=1}^{n} R e_{t}\right)\right| \geq\left|R\left(e_{t}\right)\right|$.
(a) $j \in\{2 i-1,2 i\}$.
(b) $j \in\{2 i+1\}$.
(c) $j \in\{2 i+2\}$.
(d) $j \in\{2 i+3,2 i+4\}$.
(e) $j \in\{2 i+5\}$.
(f) $j \in\{2 i+6\}$.
(g) $j \in\{2 i+7\}$.
(h) $j \in\{2 i+8\}$.
(i) $j \in\{2 i+9\}$.
(j) $j \in\{2 i+10\}$.
(k) $j \in\{2 i+10+k: 1 \leq k \leq 2 n-22\}$.

Proof:

(a) The resolving neighborhood for $1 \leq i \leq n$. When $j \in\{2 i-1\}$,

$$
R\left(u_{i}, v_{j}\right)= \begin{cases}u_{p}: & 1 \leq p \leq n, \\ v_{q}: & 1 \leq q \leq 2 n: q \neq j+1, j+2, j-2, j-3 .\end{cases}
$$

When $j \in\{2 i\}$,

$$
R\left(u_{i}, v_{j}\right)= \begin{cases}u_{p}: & 1 \leq p \leq n, \\ v_{q}: & 1 \leq q \leq 2 n, \text { where, } q \neq j+2, j+3, j-1, j-1,\end{cases}
$$

with $\left|R\left(u_{i}, v_{j}\right)\right|=3 n-4>8=\left|R\left(e_{t}\right)\right| \quad$ and $R\left(u_{i}, u_{j}\right) \cap \quad\left(\cup_{t=1}^{n} R e_{t}\right)=\left\{v_{q}: 1 \leq q \leq 2 n: \quad q \neq j+1\right.$, $j+2, j-2, j-3\}$, when $j \in\{2 i-1\}$. $R\left(u_{i}, v_{j}\right) \cap$ $\left(\cup_{t=1}^{n} \operatorname{Re}_{t}\right)=\left\{v_{q}: 1 \leq q \leq 2 n: q \neq j+2, j+3, j-1\right.$, $j-1\}$, when $j \in\{2 i\}$. Therefore, we have $\left|R\left(u_{i}, u_{j}\right) \cap\left(\cup_{t=1}^{n} R e_{t}\right)\right|=2 n-4>\left|R\left(e_{t}\right)\right|$.

(b) The resolving neighborhood for $1 \leq i \leq n, j \in\{2 i+1\}$ :

$$
R\left(u_{i}, v_{j}\right)= \begin{cases}u_{p}: & 1 \leq p \leq n, \text { where } p \neq m \text { and } j-i \leq m \leq \frac{n+1}{2}, \\ v_{q}: & 1 \leq q \leq 2 n, \text { where } q \neq j-1, s \text { where } j+3 \leq s \leq n+j-2,\end{cases}
$$

with $\quad\left|R\left(u_{i}, v_{j}\right)\right|=3 n-16>8=\left|R\left(e_{t}\right)\right| \quad$ and $R\left(u_{i}, v_{j}\right) \cap\left(\cup_{t=1}^{n} R e_{t}\right)=\left\{v_{q}: 1 \leq q \leq 2 n: \quad q \neq j-1\right.$, $s$, where, $j+3 \leq s \leq n+j-2\}$. Therefore, we have $\left|R\left(u_{i}, v_{j}\right) \cap\left(\cup_{t=1}^{n} R e_{t}\right)\right|=2 n-7>\left|R\left(e_{t}\right)\right|$. (c) The resolving neighborhood for $1 \leq i \leq n, j \in\{2 i+2\}$. When $j \in i+3$, 


$$
R\left(u_{i}, v_{j}\right)= \begin{cases}u_{p}: & 1 \leq p \leq n, \text { where } p \neq m, \text { and } j-i-1 \leq m \frac{n+1}{2}, \\ v_{q}: & 1 \leq q \leq 2 n, \text { where } q \neq s \text { and } 2(j-i-1) \leq s \leq n+j-4,\end{cases}
$$

with $\left|R\left(u_{i}, v_{j}\right)\right|=3 n-13>8=\left|R\left(e_{t}\right)\right| \quad$ and $R\left(u_{i}, v_{j}\right) \cap \quad\left(\cup_{t=1}^{n} R e_{t}\right)=\quad\left\{v_{q}: 1 \leq q \leq 2 n: q \neq s\right.$, $2(j-i-1) \leq s \leq n+j-4\}$. Therefore, we have $\left|R\left(u_{i}, v_{j}\right) \cap\left(\cup_{t=1}^{n} R e_{t}\right)\right|=2 n-8>\left|R\left(e_{t}\right)\right|$.

(d) The resolving neighborhood for $1 \leq i \leq n$, $j \in\{2 i+3,2 i+4\}:$

$R\left(u_{i}, v_{j}\right)= \begin{cases}u_{p}: & 1 \leq p \leq n, \text { where } p \neq \frac{n+1}{2}=s, \\ v_{q}: & 1 \leq q \leq 2 n, \text { where } q \neq j-2, q \neq 2 s, 2 s-1,\end{cases}$

with $\left|R\left(u_{i}, v_{j}\right)\right|=3 n-4>8=\left|R\left(e_{t}\right)\right|$ and $R\left(u_{i}, v_{j}\right) \cap$ $\left(\cup_{t=1}^{n} R e_{t}\right)=\left\{v_{q}: 1 \leq q \leq 2 n: q \neq j-2, q \neq 2 s, 2 s-1\right\}$. Therefore, we have $\left|R\left(u_{i}, v_{j}\right) \cap\left(\cup_{t=1}^{n} R e_{t}\right)\right|=$ $2 n-3>\left|R\left(e_{t}\right)\right|$.

(e) The resolving neighborhood for $1 \leq i \leq n, j \in\{2 i+5\}$ : $R\left(u_{i}, v_{j}\right)= \begin{cases}u_{p}: & 1 \leq p \leq n, \text { where } p \neq \frac{j-1}{2}, \\ v_{q}: & 1 \leq q \leq 2 n,\end{cases}$

with $\left|R\left(u_{i}, v_{j}\right)\right|=3 n-1>8=\left|R\left(e_{t}\right)\right|$ and $R\left(u_{i}, v_{j}\right) \cap\left(\cup_{t=1}^{n} R e_{t}\right)=\left\{v_{q}: 1 \leq q \leq 2 n\right\}$. Therefore, we have $\left|R\left(u_{i}, v_{j}\right) \cap\left(\cup_{t=1}^{n} R e_{t}\right)\right|=2 n>\left|R\left(e_{t}\right)\right|$.

(f) The resolving neighborhood for $1 \leq i \leq n, j \in\{2 i+6\}$ : $R\left(u_{i}, v_{j}\right)= \begin{cases}u_{p}: & 1 \leq p \leq n, \text { where } p \neq \frac{j-1}{2}, \\ v_{q}: & 1 \leq q \leq 2 n, \text { where } q \neq j-3,\end{cases}$

with $\quad\left|R\left(u_{i}, v_{j}\right)\right|=3 n-2>8=\left|R\left(e_{t}\right)\right|$ and $R\left(u_{i}, v_{j}\right) \cap\left(\cup_{t=1}^{n} R e_{t}\right)=\left\{v_{q}: 1 \leq q \leq 2 n: q \neq j-3\right\}$. Therefore, we have $\left|R\left(u_{i}, v_{j}\right) \cap\left(\cup_{t=1}^{n} R e_{t}\right)\right|=2 n-1>\left|R\left(e_{t}\right)\right|$.

(g) The resolving neighborhood for $1 \leq i \leq n$, $j \in\{2 i+3,2 i+4\}:$

$R\left(u_{i}, v_{j}\right)= \begin{cases}u_{p}: & 1 \leq p \leq n, \text { where } p \neq \frac{n+2 i+3}{2}=s, \\ v_{q}: & 1 \leq q \leq 2 n, \text { where } q \neq j-3, q \neq 2 s, 2 s-1,\end{cases}$

with $\left|R\left(u_{i}, v_{j}\right)\right|=3 n-4>8=\left|R\left(e_{t}\right)\right|$ and $R\left(u_{i}, v_{j}\right) \cap$ $\left(\cup_{t=1}^{n} R e_{t}\right)=\left\{v_{q}: 1 \leq q \leq 2 n: q \neq j-3, q \neq 2 s, 2 s-1\right\}$. Therefore, we have $\left|R\left(u_{i}, v_{j}\right) \cap\left(\cup_{t=1}^{n} R e_{t}\right)\right|=$ $2 n-3>\left|R\left(e_{t}\right)\right|$.

(h) The resolving neighborhood for $1 \leq i \leq n, j \in\{2 i+8\}$ :
$R\left(u_{i}, v_{j}\right)= \begin{cases}u_{p}: & 1 \leq p \leq n, \text { where } p \neq \frac{n+2 i+3}{2}=s, \\ v_{q}: & 1 \leq q \leq 2 n, \text { where } q \neq 2 s, 2 s-1,\end{cases}$

with $\left|R\left(u_{i}, v_{j}\right)\right|=3 n-3>8=\left|R\left(e_{t}\right)\right|$ and $R\left(u_{i}, v_{j}\right) \cap$ $\left(\cup_{t=1}^{n} R e_{t}\right)=\left\{v_{q}: 1 \leq q \leq 2 n: q \neq 2 s, 2 s-1\right\}$. Therefore, we have $\left|R\left(u_{i}, v_{j}\right) \cap\left(\cup_{t=1}^{n} R e_{t}\right)\right|=2 n-2>\left|R\left(e_{t}\right)\right|$.

(i) The resolving neighborhood for $1 \leq i \leq n, j \in\{2 i+9\}$, $R\left(u_{i}, v_{j}\right)= \begin{cases}u_{p}, & 1 \leq p \leq n, \text { where } p \neq \frac{j-3}{2}=s, \\ v_{q}, & 1 \leq q \leq 2 n, \text { where } q \neq j-4,\end{cases}$

with $\left|R\left(u_{i}, v_{j}\right)\right|=3 n-2>8=\left|R\left(e_{t}\right)\right|$ and $R\left(u_{i}, v_{j}\right) \cap\left(\cup_{t=1}^{n} R e_{t}\right)=\left\{v_{q}: 1 \leq q \leq 2 n: q \neq j-4\right\}$. Therefore, we have $\left|R\left(u_{i}, v_{j}\right) \cap\left(\cup_{t=1}^{n} R e_{t}\right)\right|=$ $2 n-1>\left|R\left(e_{t}\right)\right|$.

(j) The resolving neighborhood for $1 \leq i \leq n, j \in\{2 i+9\}$ : $R\left(u_{i}, v_{j}\right)= \begin{cases}u_{p}: & 1 \leq p \leq n, \text { where } p \neq \frac{j-4}{2}=s, \\ v_{q}: & 1 \leq q \leq 2 n, \text { where } q \neq 2 s, 2 s-1,\end{cases}$

with $\left|R\left(u_{i}, v_{j}\right)\right|=3 n-3>8=\left|R\left(e_{t}\right)\right|$ and $R\left(u_{i}, v_{j}\right) \cap$ $\left(\cup_{t=1}^{n} R e_{t}\right)=\left\{v_{q}: 1 \leq q \leq 2 n: \quad q \neq 2 s, 2 s-1\right\}$. Therefore, we have $\left|R\left(u_{i}, v_{j}\right) \cap\left(\cup_{t=1}^{n} R e_{t}\right)\right|=$ $2 n-2>\left|R\left(e_{t}\right)\right|$.

(k) The resolving neighborhood for $1 \leq i \leq n$, $j \in\{10+2 i+k: 1 \leq k \leq 2 n-22\}$.

When $k \cong 1(\bmod ) 4,2(\bmod ) 4$,

$$
R\left(u_{i}, v_{j}\right)= \begin{cases}u_{p}: & 1 \leq p \leq n, \text { where } p \neq \frac{n+7}{2}=m, \\ v_{q}: & 1 \leq q \leq 2 n, \text { where } q \neq 2 m, 2 m-1 .\end{cases}
$$

When $k \cong 0(\bmod ) 4,3(\bmod ) 4$,

$R\left(u_{i}, v_{j}\right)= \begin{cases}u_{p}: & 1 \leq p \leq n, \text { where } p \neq \frac{i+4}{2}=s, \\ v_{q}: & 1 \leq q \leq 2 n, \text { where } q \neq 2 s, 2 s-1,\end{cases}$ 
TABLE 1: FMD of metal organic graphs.

\begin{tabular}{lc}
\hline Resolving sets $(n=3)$ & Elements \\
\hline$R\left(e_{1}\right)=R\left(u_{1}, u_{2}\right)$ & $V\left(\operatorname{MOG}(3)-\left\{u_{3}, v_{5}, v_{6}\right\}\right.$ \\
$R\left(e_{2}\right)=R\left(u_{1}, u_{3}\right)$ & $V\left(\operatorname{MOG}(3)-\left\{u_{2}, v_{3}, v_{4}\right\}\right.$ \\
$R\left(e_{3}\right)=R\left(u_{1}, v_{1}\right)$ & $V\left(\operatorname{MOG}(3)-\left\{v_{2}, v_{3}, v_{5}\right\}\right.$ \\
$R\left(e_{4}\right)=R\left(u_{1}, v_{2}\right)$ & $V\left(\operatorname{MOG}(3)-\left\{v_{1}, v_{4}, v_{6}\right\}\right.$ \\
$R\left(e_{5}\right)=R\left(u_{1}, v_{3}\right)$ & $V\left(v_{5}\right\}$ \\
$R\left(e_{6}\right)=R\left(u_{1}, v_{6}\right)$ & $V(\operatorname{MOG}(3))-\left\{u_{3}, v_{1}, v_{4}\right\}$ \\
$R\left(e_{7}\right)=R\left(u_{2}, u_{3}\right)$ & $V\left(\operatorname{MOG}(3)-\left\{u_{1}, v_{1}, v_{2}\right\}\right.$ \\
$R\left(e_{8}\right)=R\left(v_{2}, v_{2}\right)$ & $V\left(\operatorname{MOG}(3)-\left\{v_{1}, v_{4}, v_{5}\right\}\right.$ \\
$R\left(e_{9}\right)=R\left(u_{2}, v_{3}\right)$ & $V\left(\operatorname{MOG}(3)-\left\{v_{2}, v_{3}, v_{6}\right\}\right.$ \\
$R\left(e_{10}\right)=R\left(u_{2}, v_{4}\right)$ & $V\left(\operatorname{MOG}(3)-\left\{u_{3}, v_{1}, v_{4}\right\}\right.$ \\
$R\left(e_{11}\right)=R\left(u_{2}, v_{5}\right)$ & $V\left(\operatorname{MOG}(3)-\left\{u_{1}, v_{3}, v_{6}\right\}\right.$ \\
$R\left(e_{12}\right)=R\left(u_{3}, v_{1}\right)$ & $V\left(\operatorname{MOG}(3)-\left\{u_{2}, v_{2}, v_{5}\right\}\right.$ \\
$R\left(e_{13}\right)=R\left(u_{3}, v_{4}\right)$ & $V\left(\operatorname{MOG}(3)-\left\{v_{1}, v_{3}, v_{6}\right\}\right.$ \\
$R\left(e_{14}\right)=R\left(u_{3}, v_{5}\right)$ & $V\left(\operatorname{MOG}(3)-\left\{v_{2}, v_{4}, v_{5}\right\}\right.$ \\
$R\left(e_{15}\right)=R\left(u_{3}, v_{6}\right)$ & $V\left(\operatorname{MOG}(3)-\left\{u_{1}, u_{2}, u_{3}\right\}\right.$ \\
$R\left(e_{16}\right)=R\left(v_{1}, v_{2}\right)$ & $V\left(\operatorname{MOG}(3)-\left\{u_{3}, v_{2}, v_{5}\right\}\right.$ \\
$R\left(e_{17}\right)=R\left(v_{1}, v_{3}\right)$ & $V\left(\operatorname{MOG}(3)-\left\{u_{2}, v_{3}, v_{6}\right\}\right.$ \\
$R\left(e_{18}\right)=R\left(v_{1}, v_{5}\right)$ & $V\left(\operatorname{MOG}(3)-\left\{u_{3}, v_{3}, v_{6}\right\}\right.$ \\
$R\left(e_{19}\right)=R\left(v_{2}, v_{4}\right)$ & $V\left(\operatorname{MOG}(3)-\left\{u_{2}, v_{1}, v_{4}\right\}\right.$ \\
$R\left(e_{20}\right)=R\left(v_{2}, v_{6}\right)$ & $V\left(\operatorname{MOG}(3)-\left\{u_{1}, u_{2}, u_{3}\right\}\right.$ \\
$R\left(e_{21}\right)=R\left(v_{3}, v_{4}\right)$ & $V\left(\operatorname{MOG}(3)-\left\{u_{1}, v_{1}, v_{4}\right\}\right.$ \\
$R\left(e_{22}\right)=R\left(v_{3}, v_{5}\right)$ & $V\left(\operatorname{MOG}(3)-\left\{u_{1}, v_{4}, v_{5}\right\}\right.$ \\
$R\left(e_{23}\right)=R\left(v_{4}, v_{6}\right)$ & $V\left(\operatorname{MOG}(3)-\left\{u_{1}, u_{2}, u_{3}\right\}\right.$ \\
$R\left(e_{24}\right)=R\left(v_{5}, v_{6}\right)$ &
\end{tabular}

TABLE 2: FMD of metal organic graphs.

\begin{tabular}{lc}
\hline Resolving sets $(n=3)$ & Elements \\
\hline$R_{1}=R\left(u_{1}, v_{4}\right)$ & $V\left(\operatorname{MOG}(3)-\left\{u_{2}, v_{6}\right\}\right.$ \\
$R_{2}=R\left(u_{1}, v_{5}\right)$ & $V\left(\operatorname{MOG}(3)-\left\{u_{3}, v_{3}\right\}\right.$ \\
$R_{3}=R\left(u_{2}, v_{1}\right)$ & $V\left(\operatorname{MOG}(3)-\left\{u_{1}, v_{5}\right\}\right.$ \\
$R_{4}=R\left(u_{2}, v_{6}\right)$ & $V$ MOG $(3)-\left\{u_{3}, v_{2}\right\}$ \\
$R_{5}=R\left(u_{3}, v_{2}\right)$ & $V\left(\operatorname{MOG}(3)-\left\{u_{1}, v_{4}\right\}\right.$ \\
$R_{6}=R\left(u_{3}, v_{3}\right)$ & $V\left(\operatorname{MOG}(3)-\left\{u_{2}, v_{1}\right\}\right.$ \\
$R_{7}=R\left(v_{1}, v_{4}\right)$ & $V\left(\operatorname{MOG}(3)-\left\{u_{3}\right\}\right.$ \\
$R_{8}=R\left(v_{1}, v_{6}\right)$ & $V\left(\operatorname{MOG}(3)-\left\{u_{5}\right\}\right.$ \\
$R_{9}=R\left(v_{2}, v_{3}\right)$ & $V M O G(3)-\left\{u_{3}\right\}$ \\
$R_{11}=R\left(v_{3}, v_{6}\right)$ & $V\left(\operatorname{MOG}(3)-\left\{u_{1}\right\}\right.$ \\
$R_{12}=R\left(v_{4}, v_{5}\right)$ & $V\left(\operatorname{MOG}(3)-\left\{u_{1}\right\}\right.$ \\
\hline
\end{tabular}

TABLE 3: FMD of metal organic graphs.

\begin{tabular}{lc}
\hline Resolving sets $(n=5)$ & Elements \\
\hline$R\left(e_{1}\right)=R\left(v_{1}, v_{2}\right)$ & $V\left(\operatorname{MOG}(5)-\left\{u_{1}, u_{2}, u_{3}, u_{4}, u_{5}, v_{6}, v_{7}\right\}\right.$ \\
$R\left(e_{1}\right)=R\left(v_{3}, v_{4}\right)$ & $V\left(\operatorname{MOG}(5)-\left\{u_{1}, u_{2}, u_{3}, u_{4}, u_{5}, v_{8}, v_{9}\right\}\right.$ \\
$R\left(e_{1}\right)=R\left(v_{5}, v_{6}\right)$ & $V\left(\operatorname{MOG}(5)-\left\{u_{1}, u_{2}, u_{3}, u_{4}, u_{5}, v_{1}, v_{10}\right\}\right.$ \\
$R\left(e_{1}\right)=R\left(v_{7}, v_{8}\right)$ & $V\left(\operatorname{MOG}(5)-\left\{u_{1}, u_{2}, u_{3}, u_{4}, u_{5}, v_{2}, v_{3}\right\}\right.$ \\
$R\left(e_{1}\right)=R\left(v_{9}, v_{10}\right)$ & $V\left(\operatorname{MOG}(5)-\left\{u_{1}, u_{2}, u_{3}, u_{4}, u_{5}, v_{4}, v_{5}\right\}\right.$ \\
\hline
\end{tabular}

with $\left|R\left(v_{i}, v_{j}\right)\right|=3 n-3>8=\left|R\left(e_{t}\right)\right|$ and $R\left(u_{i}, v_{j}\right) \cap$ $\left(\cup_{t=1}^{n} R e_{t}\right)=\left\{v_{q}: 1 \leq q \leq 2 n, q \neq 2 m, 2 m-1\right\}$, when $k \cong 1(\bmod ) 4,2(\bmod ) 4$ and $R\left(u_{i}, v_{j}\right) \cap\left(\cup_{t=1}^{n} \operatorname{Re}_{t}\right)=$ $\left\{v_{q}: 1 \leq q \leq 2 n, q \neq 2 s, 2 s-1\right\}$, when $k \cong 1(\bmod ) 4,2$ (mod)4. Therefore, we have $\left|R\left(u_{i}, v_{j}\right) \cap\left(\cup_{t=1}^{n} R e_{t}\right)\right|=$ $2 n-2>\left|R\left(e_{t}\right)\right|$.

Corollary 2. For $1 \leq i \leq n,\left|R\left(u_{i}, v_{j}\right)\right|=\left|R\left(u_{i}, v_{m}\right)\right|$, where $j \in\{2 i+s:-1 \leq s \leq 10\}$ and $m \in\{2 i+s:-2 \leq s \leq-9\}$.
TABLE 4: FMD of metal organic graphs.

\begin{tabular}{|c|c|}
\hline Resolving sets $(n=5)$ & Elements \\
\hline$R_{1}=R\left(u_{1}, u_{2}\right)$ & $V\left(\operatorname{MOG}(5)-\left\{u_{4}, v_{7}, v_{8}\right\}\right.$ \\
\hline$R_{2}=R\left(u_{1}, u_{3}\right)$ & $V\left(\operatorname{MOG}(5)-\left\{u_{2}, v_{3}, v_{4}\right\}\right.$ \\
\hline$R_{3}=R\left(u_{1}, u_{4}\right)$ & $V\left(\operatorname{MOG}(5)-\left\{u_{5}, v_{9}, v_{10}\right\}\right.$ \\
\hline$R_{4}=R\left(u_{1}, u_{5}\right)$ & $V\left(\operatorname{MOG}(5)-\left\{u_{3}, v_{5}, v_{06}\right\}\right.$ \\
\hline$R_{5}=R\left(u_{1}, v_{1}\right)$ & $V\left(\operatorname{MOG}(5)-\left\{v_{2}, v_{3}, v_{8}, v_{09}\right\}\right.$ \\
\hline$R_{6}=R\left(u_{1}, v_{2}\right)$ & $V\left(\operatorname{MOG}(5)-\left\{v_{1}, v_{4}, v_{5}, v_{10}\right\}\right.$ \\
\hline$R_{7}=R\left(u_{1}, v_{3}\right)$ & $V\left(\operatorname{MOG}(5)-\left\{u_{2}, u_{3}, v_{2}, v_{6}\right\}\right.$ \\
\hline$R_{8}=R\left(u_{1}, v_{4}\right)$ & $V\left(\operatorname{MOG}(5)-\left\{u_{2}, u_{3}, v_{7}\right\}\right.$ \\
\hline$R_{9}=R\left(u_{1}, v_{5}\right)$ & $V\left(\operatorname{MOG}(5)-\left\{u_{4}, v_{3}, v_{8}\right\}\right.$ \\
\hline$R_{10}=R\left(u_{1}, v_{6}\right)$ & $V\left(\operatorname{MOG}(5)-\left\{u_{4}, v_{4}\right\}\right.$ \\
\hline$R_{11}=R\left(u_{1}, v_{7}\right)$ & $V\left(\operatorname{MOG}(5)-\left\{u_{3}, v_{9}\right\}\right.$ \\
\hline$R_{12}=R\left(u_{1}, v_{8}\right)$ & $V\left(\operatorname{MOG}(5)-\left\{u_{3}, v_{5}, v_{10}\right\}\right.$ \\
\hline$R_{13}=R\left(u_{1}, v_{9}\right)$ & $V\left(\operatorname{MOG}(5)-\left\{u_{4}, u_{5}, v_{6}\right\}\right.$ \\
\hline$R_{14}=R\left(u_{1}, v_{10}\right)$ & $V\left(\operatorname{MOG}(5)-\left\{u_{4}, u_{5}, v_{1}, v_{7}\right\}\right.$ \\
\hline$R_{15}=R\left(u_{2}, u_{3}\right)$ & $V\left(\operatorname{MOG}(5)-\left\{u_{5}, v_{9}, v_{10}\right\}\right.$ \\
\hline$R_{16}=R\left(u_{2}, u_{4}\right)$ & $V\left(\operatorname{MOG}(5)-\left\{u_{3}, v_{5}, v_{6}\right\}\right.$ \\
\hline$R_{17}=R\left(u_{2}, u_{5}\right)$ & $V\left(\operatorname{MOG}(5)-\left\{u_{1}, v_{1}, v_{2}\right\}\right.$ \\
\hline$R_{18}=R\left(u_{2}, v_{1}\right)$ & $V\left(\operatorname{MOG}(5)-\left\{u_{1}, u_{5}, v_{8}\right\}\right.$ \\
\hline$R_{19}=R\left(u_{2}, v_{2}\right)$ & $V\left(\operatorname{MOG}(5)-\left\{u_{1}, u_{5}, v_{9}\right\}\right.$ \\
\hline$R_{20}=R\left(u_{2}, v_{3}\right)$ & $V\left(\operatorname{MOG}(5)-\left\{v_{1}, v_{4}, v_{5}, v_{10}\right\}\right.$ \\
\hline$R_{21}=R\left(u_{2}, v_{4}\right)$ & $V\left(\operatorname{MOG}(5)-\left\{v_{2}, v_{3}, v_{6}, v_{7}\right\}\right.$ \\
\hline$R_{22}=R\left(u_{2}, v_{5}\right)$ & $V\left(\operatorname{MOG}(5)-\left\{u_{3}, u_{4}, v_{4}, v_{8}\right\}\right.$ \\
\hline$R_{23}=R\left(u_{2}, v_{6}\right)$ & $V\left(\operatorname{MOG}(5)-\left\{u_{3}, u_{4}, v_{9}\right\}\right.$ \\
\hline$R_{24}=R\left(u_{2}, v_{7}\right)$ & $V\left(\operatorname{MOG}(5)-\left\{u_{5}, v_{5}, v_{10}\right\}\right.$ \\
\hline$R_{25}=R\left(u_{2}, v_{8}\right)$ & $V\left(\operatorname{MOG}(5)-\left\{u_{5}, v_{6}\right\}\right.$ \\
\hline$R_{26}=R\left(u_{2}, v_{9}\right)$ & $V\left(\operatorname{MOG}(5)-\left\{u_{4}, v_{1}\right\}\right.$ \\
\hline$R_{27}=R\left(u_{2}, v_{10}\right)$ & $V\left(\operatorname{MOG}(5)-\left\{u_{4}, v_{2}, v_{7}\right\}\right.$ \\
\hline$R_{28}=R\left(u_{3}, u_{4}\right)$ & $V\left(\operatorname{MOG}(5)-\left\{u_{1}, v_{1}, v_{2}\right\}\right.$ \\
\hline$R_{29}=R\left(u_{3}, u_{5}\right)$ & $V\left(\operatorname{MOG}(5)-\left\{u_{4}, v_{7}, v_{8}\right\}\right.$ \\
\hline$R_{30}=R\left(u_{3}, v_{1}\right)$ & $V\left(\operatorname{MOG}(5)-\left\{u_{5}, v_{3}\right\}\right.$ \\
\hline$R_{31}=R\left(u_{3}, v_{2}\right)$ & $V\left(\operatorname{MOG}(5)-\left\{u_{5}, v_{4}, v_{9}\right\}\right.$ \\
\hline$R_{32}=R\left(u_{3}, v_{3}\right)$ & $V\left(\operatorname{MOG}(5)-\left\{u_{1}, u_{2}, v_{10}\right\}\right.$ \\
\hline$R_{33}=R\left(u_{3}, v_{4}\right)$ & $V\left(\operatorname{MOG}(5)-\left\{u_{1}, u_{2}, v_{1}, v_{5}\right\}\right.$ \\
\hline$R_{34}=R\left(u_{3}, v_{5}\right)$ & $V\left(\operatorname{MOG}(5)-\left\{v_{2}, v_{3}, v_{6}, v_{7}\right\}\right.$ \\
\hline$R_{35}=R\left(u_{3}, v_{6}\right)$ & $V\left(\operatorname{MOG}(5)-\left\{v_{4}, v_{5}, v_{8}, v_{9}\right\}\right.$ \\
\hline$R_{36}=R\left(u_{3}, v_{7}\right)$ & $V\left(\operatorname{MOG}(5)-\left\{u_{4}, u_{5}, v_{6}, v_{10}\right\}\right.$ \\
\hline$R_{37}=R\left(u_{3}, v_{8}\right)$ & $V\left(\operatorname{MOG}(5)-\left\{u_{4}, u_{5}, v_{1}\right\}\right.$ \\
\hline$R_{38}=R\left(u_{3}, v_{9}\right)$ & $V\left(\operatorname{MOG}(5)-\left\{u_{1}, v_{2}, v_{7}\right\}\right.$ \\
\hline$R_{39}=R\left(u_{3}, v_{10}\right)$ & $V\left(\operatorname{MOG}(5)-\left\{u_{1}, v_{8}\right\}\right.$ \\
\hline$R_{40}=R\left(u_{4}, u_{5}\right)$ & $V\left(\operatorname{MOG}(5)-\left\{u_{2}, v_{3}, v_{4}\right\}\right.$ \\
\hline$R_{41}=R\left(u_{4}, v_{1}\right)$ & $V\left(\operatorname{MOG}(5)-\left\{u_{2}, v_{4}, v_{9}\right\}\right.$ \\
\hline$R_{42}=R\left(u_{4}, v_{2}\right)$ & $V\left(\operatorname{MOG}(5)-\left\{u_{2}, v_{10}\right\}\right.$ \\
\hline$R_{43}=R\left(u_{4}, v_{3}\right)$ & $V\left(\operatorname{MOG}(5)-\left\{u_{1}, v_{5}\right\}\right.$ \\
\hline$R_{44}=R\left(u_{4}, v_{4}\right)$ & $V\left(\operatorname{MOG}(5)-\left\{u_{1}, v_{1}, v_{6}\right\}\right.$ \\
\hline$R_{45}=R\left(u_{4}, v_{5}\right)$ & $V\left(\operatorname{MOG}(5)-\left\{u_{2}, u_{3}, v_{2}\right\}\right.$ \\
\hline$R_{46}=R\left(u_{4}, v_{6}\right)$ & $V\left(\operatorname{MOG}(5)-\left\{u_{2}, u_{3}, v_{2}, v_{7}\right\}\right.$ \\
\hline$R_{47}=R\left(u_{4}, v_{7}\right)$ & $V\left(\operatorname{MOG}(5)-\left\{v_{4}, v_{5}, v_{8}, v_{9}\right\}\right.$ \\
\hline$R_{48}=R\left(u_{4}, v_{8}\right)$ & $V\left(\operatorname{MOG}(5)-\left\{v_{1}, v_{6}, v_{7}, v_{10}\right\}\right.$ \\
\hline$R_{49}=R\left(u_{4}, v_{9}\right)$ & $V\left(\operatorname{MOG}(5)-\left\{u_{1}, u_{5}, v_{2}, v_{8}\right\}\right.$ \\
\hline$R_{50}=R\left(u_{4}, v_{10}\right)$ & $V\left(\operatorname{MOG}(5)-\left\{u_{1}, u_{5}, v_{3}\right\}\right.$ \\
\hline$R_{51}=R\left(u_{5}, v_{1}\right)$ & $V\left(\operatorname{MOG}(5)-\left\{u_{1}, u_{2}, v_{4}, v_{10}\right\}\right.$ \\
\hline$R_{52}=R\left(u_{5}, v_{2}\right)$ & $V\left(\operatorname{MOG}(5)-\left\{u_{1}, u_{2}, v_{5}\right\}\right.$ \\
\hline$R_{53}=R\left(u_{5}, v_{3}\right)$ & $V\left(\operatorname{MOG}(5)-\left\{u_{3}, v_{1}, v_{6}\right\}\right.$ \\
\hline$R_{54}=R\left(u_{5}, v_{4}\right)$ & $V\left(\operatorname{MOG}(5)-\left\{u_{3}, v_{2}\right\}\right.$ \\
\hline$R_{55}=R\left(u_{5}, v_{5}\right)$ & $V\left(\operatorname{MOG}(5)-\left\{u_{2}, v_{7}\right\}\right.$ \\
\hline$R_{56}=R\left(u_{5}, v_{6}\right)$ & $V\left(\operatorname{MOG}(5)-\left\{u_{2}, v_{3}, v_{8}\right\}\right.$ \\
\hline$R_{57}=R\left(u_{5}, v_{7}\right)$ & $V\left(\operatorname{MOG}(5)-\left\{u_{3}, u_{4}, v_{4}\right\}\right.$ \\
\hline$R_{58}=R\left(u_{5}, v_{8}\right)$ & $V\left(\operatorname{MOG}(5)-\left\{u_{3}, u_{4}, v_{5}, v_{9}\right\}\right.$ \\
\hline$R_{59}=R\left(u_{5}, v_{9}\right)$ & $V\left(\operatorname{MOG}(5)-\left\{v_{1}, v_{6}, v_{7}, v_{9}\right\}\right.$ \\
\hline$R_{60}=R\left(u_{5}, v_{10}\right)$ & $V\left(\operatorname{MOG}(5)-\left\{v_{2}, v_{3}, v_{8}, v_{9}\right\}\right.$ \\
\hline
\end{tabular}


TABLE 4: Continued.

\begin{tabular}{|c|c|}
\hline Resolving sets $(n=5)$ & Elements \\
\hline$R_{61}=R\left(v_{01}, v_{03}\right)$ & $V\left(\operatorname{MOG}(5)-\left\{u_{4}, v_{2}, v_{7}\right\}\right.$ \\
\hline$R_{62}=R\left(v_{01}, v_{04}\right)$ & $V\left(\operatorname{MOG}(5)-\left\{u_{4}\right\}\right.$ \\
\hline$R_{63}=R\left(v_{01}, v_{05}\right)$ & $V\left(\operatorname{MOG}(5)-\left\{u_{2}, v_{3}, v_{8}\right\}\right.$ \\
\hline$R_{64}=R\left(v_{01}, v_{06}\right)$ & $V\left(\operatorname{MOG}(5)-\left\{u_{2}\right\}\right.$ \\
\hline$R_{65}=R\left(v_{01}, v_{07}\right)$ & $V\left(\operatorname{MOG}(5)-\left\{u_{5}, v_{4}, v_{9}\right\}\right.$ \\
\hline$R_{66}=R\left(v_{01}, v_{08}\right)$ & $V\left(\operatorname{MOG}(5)-\left\{u_{5}\right\}\right.$ \\
\hline$R_{67}=R\left(v_{01}, v_{09}\right)$ & $V\left(\operatorname{MOG}(5)-\left\{u_{3}, v_{5}\right\}\right.$ \\
\hline$R_{68}=R\left(v_{01}, v_{10}\right)$ & $V\left(\operatorname{MOG}(5)-\left\{u_{3}, v_{5}, v_{6}\right\}\right.$ \\
\hline$R_{69}=R\left(v_{02}, v_{03}\right)$ & $V\left(\operatorname{MOG}(5)-\left\{u_{4}, v_{7}, v_{8}\right\}\right.$ \\
\hline$R_{70}=R\left(v_{02}, v_{04}\right)$ & $V\left(\operatorname{MOG}(5)-\left\{u_{4}, v_{3}, v_{8}\right\}\right.$ \\
\hline$R_{71}=R\left(v_{02}, v_{05}\right)$ & $V\left(\operatorname{MOG}(5)-\left\{u_{2}\right\}\right.$ \\
\hline$R_{72}=R\left(v_{02}, v_{06}\right)$ & $V\left(\operatorname{MOG}(5)-\left\{u_{2}, v_{4}, v_{9}\right\}\right.$ \\
\hline$R_{73}=R\left(v_{02}, v_{07}\right)$ & $V\left(\operatorname{MOG}(5)-\left\{u_{5}\right\}\right.$ \\
\hline$R_{74}=R\left(v_{02}, v_{08}\right)$ & $V\left(\operatorname{MOG}(5)-\left\{u_{5}, v_{5}, v_{10}\right\}\right.$ \\
\hline$R_{75}=R\left(v_{02}, v_{09}\right)$ & $V\left(\operatorname{MOG}(5)-\left\{u_{6}\right\}\right.$ \\
\hline$R_{76}=R\left(v_{02}, v_{10}\right)$ & $V\left(\operatorname{MOG}(5)-\left\{u_{3}, v_{1}, v_{6}\right\}\right.$ \\
\hline$R_{77}=R\left(v_{03}, v_{05}\right)$ & $V\left(\operatorname{MOG}(5)-\left\{u_{5}, v_{4}, v_{9}\right\}\right.$ \\
\hline$R_{78}=R\left(v_{03}, v_{06}\right)$ & $V\left(\operatorname{MOG}(5)-\left\{u_{5}\right\}\right.$ \\
\hline$R_{79}=R\left(v_{03}, v_{07}\right)$ & $V\left(\operatorname{MOG}(5)-\left\{u_{3}, v_{5}, v_{10}\right\}\right.$ \\
\hline$R_{80}=R\left(v_{03}, v_{08}\right)$ & $V\left(\operatorname{MOG}(5)-\left\{u_{3}\right\}\right.$ \\
\hline$R_{81}=R\left(v_{03}, v_{09}\right)$ & $V\left(\operatorname{MOG}(5)-\left\{u_{1}, v_{1}, v_{6}\right\}\right.$ \\
\hline$R_{82}=R\left(v_{03}, v_{10}\right)$ & $V\left(\operatorname{MOG}(5)-\left\{u_{1}\right\}\right.$ \\
\hline$R_{83}=R\left(v_{04}, v_{05}\right)$ & $V\left(\operatorname{MOG}(5)-\left\{u_{5}, v_{9}, v_{10}\right\}\right.$ \\
\hline$R_{84}=R\left(v_{04}, v_{06}\right)$ & $V\left(\operatorname{MOG}(5)-\left\{u_{5}, v_{5}, v_{10}\right\}\right.$ \\
\hline$R_{85}=R\left(v_{04}, v_{07}\right)$ & $V\left(\operatorname{MOG}(5)-\left\{u_{3}\right\}\right.$ \\
\hline$R_{86}=R\left(v_{04}, v_{08}\right)$ & $V\left(\operatorname{MOG}(5)-\left\{u_{3}, v_{1}, v_{6}\right\}\right.$ \\
\hline$R_{87}=R\left(v_{04}, v_{09}\right)$ & $V\left(\operatorname{MOG}(5)-\left\{u_{1}\right\}\right.$ \\
\hline$R_{88}=R\left(v_{04}, v_{10}\right)$ & $V\left(\operatorname{MOG}(5)-\left\{u_{1}, v_{2}, v_{7}\right\}\right.$ \\
\hline$R_{89}=R\left(v_{05}, v_{07}\right)$ & $V\left(\operatorname{MOG}(5)-\left\{u_{1}, v_{1}, v_{6}\right\}\right.$ \\
\hline$R_{90}=R\left(v_{05}, v_{08}\right)$ & $V\left(\operatorname{MOG}(5)-\left\{u_{1}\right\}\right.$ \\
\hline$R_{91}=R\left(v_{05}, v_{09}\right)$ & $V\left(\operatorname{MOG}(5)-\left\{u_{4}, v_{4} 2, v_{7}\right\}\right.$ \\
\hline$R_{92}=R\left(v_{05}, v_{10}\right)$ & $V\left(\operatorname{MOG}(5)-\left\{u_{4}\right\}\right.$ \\
\hline$R_{93}=R\left(v_{06}, v_{07}\right)$ & $V\left(\operatorname{MOG}(5)-\left\{u_{1}, v_{1}, v_{2}\right\}\right.$ \\
\hline$R_{94}=R\left(v_{06}, v_{08}\right)$ & $V\left(\operatorname{MOG}(5)-\left\{u_{1}, v_{2}, v_{7}\right\}\right.$ \\
\hline$R_{95}=R\left(v_{06}, v_{09}\right)$ & $V\left(\operatorname{MOG}(5)-\left\{u_{4}\right\}\right.$ \\
\hline$R_{96}=R\left(v_{06}, v_{10}\right)$ & $V\left(\operatorname{MOG}(5)-\left\{u_{4}, v_{3}, v_{8}\right\}\right.$ \\
\hline$R_{97}=R\left(v_{07}, v_{09}\right)$ & $V\left(\operatorname{MOG}(5)-\left\{u_{2}, v_{3}, v_{8}\right\}\right.$ \\
\hline$R_{98}=R\left(v_{07}, v_{10}\right)$ & $V\left(\operatorname{MOG}(5)-\left\{u_{2}\right\}\right.$ \\
\hline$R_{99}=R\left(v_{08}, v_{09}\right)$ & $V\left(\operatorname{MOG}(5)-\left\{u_{2}, v_{3}, v_{4}\right\}\right.$ \\
\hline$R_{100}=R\left(v_{08}, v_{10}\right)$ & $V\left(\operatorname{MOG}(5)-\left\{u_{2}, v_{4}, v_{9}\right\}\right.$ \\
\hline
\end{tabular}

Theorem 2. The FMD of metal organic graph $M O G(n)$ for $3 \leq n \leq 7, n \cong 1(\bmod 2)$ is

$$
\operatorname{dim}_{f} \operatorname{MOG}(n)= \begin{cases}\frac{3}{2} & \text { if } n=3 \\ \frac{5}{4} & \text { if } n=5 \\ \frac{7}{4} & \text { if } n=7\end{cases}
$$

Proof. Case 1: when $n=3$, then the RNs are as follows.

Since, for $1 \leq t \leq 24$, the cardinality of each RN $R\left(e_{t}\right)$ is 6 , as given in Table 1 , which is less than the cardinalities of all other RNs $R_{m}$ of MOG(3), as given in Table 2, where $1 \leq m \leq 12$. Moreover, $\cup_{t=1}^{24} R\left(e_{t}\right)=V(\operatorname{MOG}(3)$;
TABle 5: FMD of metal organic graphs.

\begin{tabular}{lc}
\hline Resolving sets $(n=5)$ & Elements \\
\hline$R\left(e_{1}\right)=R\left(v_{1}, v_{2}\right)$ & $\left\{v_{1}, v_{2}, v_{3}, v_{4}, v_{5}, v_{12}, v_{13}, v_{14}\right\}$ \\
$R\left(e_{2}\right)=R\left(v_{3}, v_{4}\right)$ & $\left\{v_{1}, v_{2}, v_{3}, v_{4}, v_{5}, v_{6}, v_{7}, v_{14}\right\}$ \\
$R\left(e_{3}\right)=R\left(v_{5}, v_{6}\right)$ & $\left\{v_{2}, v_{3}, v_{4}, v_{5}, v_{6}, v_{7}, v_{8}, v_{9}\right\}$ \\
$R\left(e_{4}\right) R\left(v_{7}, v_{8}\right)$ & $\left\{v_{4}, v_{5}, v_{6}, v_{7}, v_{8}, v_{9}, v_{10}, v_{11}\right\}$ \\
$R\left(e_{5}\right)=R\left(v_{9}, v_{10}\right)$ & $\left\{v_{6}, v_{7}, v_{8}, v_{9}, v_{10}, v_{11}, v_{12}, v_{13}\right\}$ \\
$R\left(e_{6}\right)=R\left(v_{11}, v_{12}\right)$ & $\left\{v_{1}, v_{8}, v_{9}, v_{10}, v_{11}, v_{12}, v_{13}, v_{14}\right\}$ \\
$R\left(e_{7}\right)=R\left(v_{13}, v_{14}\right)$ & $\left\{v_{1}, v_{2}, v_{3}, v_{10}, v_{11}, v_{12}, v_{13}, v_{14}\right\}$ \\
\hline
\end{tabular}

this implies that $\cup_{t=1}^{24} R\left(e_{t}\right)=9$ and $\left|R_{m} \cap \cup_{t=1}^{24} R\left(e_{t}\right)\right|>$ $R\left(e_{t}\right) \mid=6$.

Consequently, $\operatorname{dim}_{f}\left(\operatorname{MOG}(3)=\sum_{t=1}^{9} 1 / 6 \leq 3 / 2\right.$.

Case 2: when $n=5$, as shown in Figure 1, the RNs are as follows.

Since, for $1 \leq t \leq 5$, the cardinality of each RN $R\left(e_{t}\right)$ is 8 , as given in Table 3 , which is less than the cardinalities of all other RNs $R_{m}$ of $(\operatorname{MOG}(5)$, as given in Table 4 , where $\quad 1 \leq m \leq 100$. Moreover, $\quad \cup_{t=1}^{5} R\left(e_{t}\right)=$ $V\left(\left(\operatorname{MOG}(5)\right.\right.$; this implies that $\left|\cup_{t=1}^{5} R\left(e_{t}\right)\right|=10$ and $R_{m} \cap\left|\cup_{t=1}^{5} R\left(e_{t}\right)\right|>R\left(e_{t}\right) \mid=8$.

Consequently, $\operatorname{dim}_{f}\left((M O G(5)) \leq \sum_{t=1}^{10} 1 / 8=5 / 4\right.$.

Case 3: when $n=7$, as shown in Figure 1, then the RNs are as follows.

Since, for $1 \leq t \leq 7$, the cardinality of each $\mathrm{RN} R\left(e_{t}\right)$ is 8 , as given in Table 5, which is less than the cardinalities of all other RNs $R_{m}$ of (MOG(5)), as given in Table 6, where $1 \leq m \leq 203$. Moreover, $\cup_{t=1}^{7} R\left(e_{t}\right)=V(\operatorname{MOG}(7)$; this implies that $\left|\cup_{t=1}^{7} R\left(e_{t}\right)\right|=14$ and $\left|R_{m} \cap \cup_{t=1}^{7} R\left(e_{t}\right)\right|>$ $R\left(e_{t}\right) \mid=8$.

Consequently, $\operatorname{dim}_{f}\left(\operatorname{MOG}(5) \leq \sum_{t=1}^{14} 1 / 8 \leq 7 / 4\right.$.

Theorem 3. Let $M O G(n)$ for $n \geq 9$ and $n \cong 1(\bmod ) 2$ be the metal organic graph. Then, $\operatorname{dim}_{l f} M O G(n) \leq n / 4$.

Proof. In view of Lemmas $3-8$ for $1 \leq k \leq n, j=i+1$, $i \in[2 k-1], \quad 1 \leq t \leq n, \quad\left|R\left(e_{t}\right)\right|=\left|R\left(v_{i} v_{j}\right)\right|=8 \quad$ and $|X|=\left|\cup_{t=1}^{n} R\left(e_{t}\right)\right|=2 n$. Also, we have $|R(x y)| \leq\left|R\left(e_{t}\right)\right|$ for all $x y \in E(\operatorname{MOG}(n))$. Moreover, the local resolving neighbourhood of minimum cardinality is not disjoint. Therefore, the fractional metric of $\operatorname{MOG}(n)$ is given as follows:

$$
\operatorname{dim}_{f} \operatorname{MOG}(n) \leq \sum_{t=1}^{|X|} \frac{1}{\left|R\left(e_{t}\right)\right|} .
$$

For $|X|=2 n$ and $\left|R\left(e_{t}\right)\right|=8$, we have

$$
\operatorname{dim}_{f} \operatorname{MOG}(n) \leq \sum_{t=1}^{2 n} \frac{1}{8} .
$$

Hence, $\operatorname{dim}_{f} \operatorname{MOG}(n) \leq n / 4$. 
TABLe 6: FMD of MOG(7).

\begin{tabular}{|c|c|}
\hline Resolving sets $(n=7)$ & Elements \\
\hline$R_{1}=R\left(u_{1}, u_{2}\right)$ & $V\left(\operatorname{MOG}(7)-\left\{u_{5}, v_{9}, v_{10}\right\}\right.$ \\
\hline$R_{2}=R\left(u_{1}, u_{3}\right)$ & $V\left(\operatorname{MOG}(7)-\left\{u_{2}, v_{3}, v_{4}\right\}\right.$ \\
\hline$R_{3}=R\left(u_{1}, u_{4}\right)$ & $V\left(\operatorname{MOG}(7)-\left\{u_{6}, v_{11}, v_{12}\right\}\right.$ \\
\hline$R_{4}=R\left(u_{1}, u_{5}\right)$ & $V\left(\operatorname{MOG}(7)-\left\{u_{3}, v_{5}, v_{06}\right\}\right.$ \\
\hline$R_{5}=R\left(u_{1}, u_{6}\right)$ & $V\left(\operatorname{MOG}(7)-\left\{u_{7}, v_{13}, v_{14}\right\}\right.$ \\
\hline$R_{6}=R\left(u_{1}, u_{7}\right)$ & $V\left(\operatorname{MOG}(7)-\left\{u_{4}, v_{7}, v_{08}\right\}\right.$ \\
\hline$R_{7}=R\left(u_{1}, v_{1}\right)$ & $V\left(\operatorname{MOG}(7)-\left\{v_{2}, v_{3}, v_{12}, v_{13}\right\}\right.$ \\
\hline$R_{8}=R\left(u_{1}, v_{2}\right)$ & $V\left(\operatorname{MOG}(7)-\left\{v_{1}, v_{4}, v_{5}, v_{14}\right\}\right.$ \\
\hline$R_{9}=R\left(u_{1}, v_{3}\right)$ & $V\left(\operatorname{MOG}(7)-\left\{u_{2}, u_{3}, u_{4}, v_{2}, v_{6}, v_{7}, v_{8}\right\}\right.$ \\
\hline$R_{10}=R\left(u_{1}, v_{4}\right)$ & $V\left(\operatorname{MOG}(7)-\left\{u_{2}, u_{3}, u_{4}, v_{8}\right\}\right.$ \\
\hline$R_{11}=R\left(u_{1}, v_{5}\right)$ & $V\left(\operatorname{MOG}(7)-\left\{u_{5}, v_{3}, v_{9}, v_{10}\right\}\right.$ \\
\hline$R_{12}=R\left(u_{1}, v_{6}\right)$ & $V\left(\operatorname{MOG}(7)-\left\{u_{5}, v_{4}, v_{10}\right\}\right.$ \\
\hline$R_{13}=R\left(u_{1}, v_{7}\right)$ & $V\left(\operatorname{MOG}(7)-\left\{u_{3}\right\}\right.$ \\
\hline$R_{14}=R\left(u_{1}, v_{8}\right)$ & $V\left(\operatorname{MOG}(7)-\left\{u_{3}, v_{5}, v_{11}\right\}\right.$ \\
\hline$R_{15}=R\left(u_{1}, v_{9}\right)$ & $V\left(\operatorname{MOG}(7)-\left\{u_{6}, v_{6}, v_{12}\right\}\right.$ \\
\hline$R_{16}=R\left(u_{1}, v_{10}\right)$ & $V\left(\operatorname{MOG}(7)-\left\{u_{6}\right\}\right.$ \\
\hline$R_{17}=R\left(u_{1}, v_{11}\right)$ & $V\left(\operatorname{MOG}(7)-\left\{u_{4}, v_{7}\right\}\right.$ \\
\hline$R_{18}=R\left(u_{1}, v_{12}\right)$ & $V\left(\operatorname{MOG}(7)-\left\{u_{4}, v_{7}, v_{8}, v_{14}\right\}\right.$ \\
\hline$R_{19}=R\left(u_{1}, v_{13}\right)$ & $V\left(\operatorname{MOG}(7)-\left\{u_{5}, u_{6}, u_{7}, v_{9}\right\}\right.$ \\
\hline$R_{20}=R\left(u_{1}, v_{14}\right)$ & $V\left(\operatorname{MOG}(7)-\left\{u_{5}, u_{6}, u_{7}, v_{1}, v_{9}\right\}\right.$ \\
\hline$R_{21}=R\left(u_{2}, u_{3}\right)$ & $V\left(\operatorname{MOG}(7)-\left\{u_{6}, v_{11}, v_{12}\right\}\right.$ \\
\hline$R_{22}=R\left(u_{2}, u_{4}\right)$ & $V\left(\operatorname{MOG}(7)-\left\{u_{3}, v_{5}, v_{6}\right\}\right.$ \\
\hline$R_{23}=R\left(u_{2}, u_{5}\right)$ & $V\left(\operatorname{MOG}(7)-\left\{u_{7}, v_{13}, v_{14}\right\}\right.$ \\
\hline$R_{24}=R\left(u_{2}, u_{6}\right)$ & $V\left(\operatorname{MOG}(7)-\left\{u_{4}, v_{7}, v_{8}\right\}\right.$ \\
\hline$R_{25}=R\left(u_{2}, u_{7}\right)$ & $V\left(\operatorname{MOG}(7)-\left\{u_{1}, v_{1}, v_{2},\right\}\right.$ \\
\hline$R_{26}=R\left(u_{2}, v_{1}\right)$ & $V\left(\operatorname{MOG}(7)-\left\{u_{1}, u_{6}, u_{7}, v_{11}\right\}\right.$ \\
\hline$R_{27}=R\left(u_{2}, v_{2}\right)$ & $V\left(\operatorname{MOG}(7)-\left\{u_{1}, u_{6}, u_{7}, v_{3}, u_{11}, v_{12}, v_{13}\right\}\right.$ \\
\hline$R_{28}=R\left(u_{2}, v_{3}\right)$ & $V\left(\operatorname{MOG}(7)-\left\{v_{1}, v_{4}, v_{5}, v_{14}\right\}\right.$ \\
\hline$R_{29}=R\left(u_{2}, v_{4}\right)$ & $V\left(\operatorname{MOG}(7)-\left\{v_{2}, v_{3}, v_{6}, v_{7}\right\}\right.$ \\
\hline$R_{30}=R\left(u_{2}, v_{5}\right)$ & $V\left(\operatorname{MOG}(7)-\left\{u_{3}, u_{4}, u_{5}, v_{4}, v_{8}, v_{9}, v_{10}\right\}\right.$ \\
\hline$R_{31}=R\left(u_{2}, v_{6}\right)$ & $V\left(\operatorname{MOG}(7)-\left\{u_{3}, u_{4}, u_{5}, v_{10}\right\}\right.$ \\
\hline$R_{32}=R\left(u_{2}, v_{7}\right)$ & $V\left(\operatorname{MOG}(7)-\left\{u_{6}, v_{5}, v_{11}, v_{12}\right\}\right.$ \\
\hline$R_{33}=R\left(u_{2}, v_{8}\right)$ & $V\left(\operatorname{MOG}(7)-\left\{u_{6}, v_{6}, v_{12}\right\}\right.$ \\
\hline$R_{34}=R\left(u_{2}, v_{9}\right)$ & $V\left(\operatorname{MOG}(7)-\left\{u_{4}\right\}\right.$ \\
\hline$R_{35}=R\left(u_{2}, v_{10}\right)$ & $V\left(\operatorname{MOG}(7)-\left\{u_{4}, v_{7}, v_{13}\right\}\right.$ \\
\hline$R_{36}=R\left(u_{2}, v_{11}\right)$ & $V\left(\operatorname{MOG}(7)-\left\{v_{8}, v_{14}\right\}\right.$ \\
\hline$R_{37}=R\left(u_{2}, v_{12}\right)$ & $V\left(\operatorname{MOG}(7)-\left\{u_{7}\right\}\right.$ \\
\hline$R_{38}=R\left(u_{2}, v_{13}\right)$ & $V\left(\operatorname{MOG}(7)-\left\{u_{5}, v_{1}, v_{9}\right\}\right.$ \\
\hline$R_{39}=R\left(u_{2}, v_{14}\right)$ & $V\left(\operatorname{MOG}(7)-\left\{u_{5}, v_{2}, v_{9}, v_{10}\right\}\right.$ \\
\hline$R_{40}=R\left(u_{3}, u_{4}\right)$ & $V\left(\operatorname{MOG}(7)-\left\{u_{7}, v_{13}, v_{14}\right\}\right.$ \\
\hline$R_{41}=R\left(u_{3}, u_{5}\right)$ & $V\left(\operatorname{MOG}(7)-\left\{u_{4}, v_{7}, v_{8}\right\}\right.$ \\
\hline$R_{42}=R\left(u_{3}, u_{6}\right)$ & $V\left(\operatorname{MOG}(7)-\left\{u_{1}, v_{1}, v_{2}\right\}\right.$ \\
\hline$R_{43}=R\left(u_{3}, u_{7}\right)$ & $V\left(\operatorname{MOG}(7)-\left\{u_{5}, v_{9}, v_{10}\right\}\right.$ \\
\hline$R_{44}=R\left(u_{3}, v_{1}\right)$ & $V\left(\operatorname{MOG}(7)-\left\{u_{6}, v_{3}, v_{11}\right\}\right.$ \\
\hline$R_{45}=R\left(u_{3}, v_{2}\right)$ & $V\left(\operatorname{MOG}(7)-\left\{u_{6}, v_{4}, v_{11}, v_{12}\right\}\right.$ \\
\hline$R_{46}=R\left(u_{3}, v_{3}\right)$ & $V\left(\operatorname{MOG}(7)-\left\{u_{1}, u_{2}, u_{7}, v_{13}, v_{14}\right\}\right.$ \\
\hline$R_{47}=R\left(u_{3}, v_{4}\right)$ & $V\left(\operatorname{MOG}(7)-\left\{u_{1}, u_{2}, u_{7}, v_{1}, v_{5}, v_{13}, v_{14}\right\}\right.$ \\
\hline$R_{48}=R\left(u_{3}, v_{5}\right)$ & $V\left(\operatorname{MOG}(7)-\left\{v_{2}, v_{3}, v_{6}, v_{7}\right\}\right.$ \\
\hline$R_{49}=R\left(u_{3}, v_{6}\right)$ & $V\left(\operatorname{MOG}(7)-\left\{v_{4}, v_{5}, v_{8}, v_{9}\right\}\right.$ \\
\hline$R_{50}=R\left(u_{3}, v_{7}\right)$ & $V\left(\operatorname{MOG}(7)-\left\{u_{4}, u_{5}, u_{6}, v_{6}, v_{10}, v_{11}, v_{12}\right\}\right.$ \\
\hline$R_{51}=R\left(u_{3}, v_{8}\right)$ & $V\left(\operatorname{MOG}(7)-\left\{u_{4}, u_{5}, u_{6}, v_{12}\right\}\right.$ \\
\hline$R_{52}=R\left(u_{3}, v_{9}\right)$ & $V\left(\operatorname{MOG}(7)-\left\{u_{7}, v_{7}, v_{13}\right\}\right.$ \\
\hline$R_{53}=R\left(u_{3}, v_{10}\right)$ & $V\left(\operatorname{MOG}(7)-\left\{u_{7}, v_{8}, v_{14}\right\}\right.$ \\
\hline$R_{54}=R\left(u_{3}, v_{11}\right)$ & $V\left(\operatorname{MOG}(7)-\left\{u_{5}\right\}\right.$ \\
\hline$R_{55}=R\left(u_{3}, v_{12}\right)$ & $V\left(\operatorname{MOG}(7)-\left\{u_{5}, v_{1}\right\}\right.$ \\
\hline$R_{56}=R\left(u_{3}, v_{13}\right)$ & $V\left(\operatorname{MOG}(7)-\left\{u_{1}, u_{2}, v_{10}\right\}\right.$ \\
\hline$R_{57}=R\left(u_{3}, v_{14}\right)$ & $V\left(\operatorname{MOG}(7)-\left\{u_{1}, v_{10}\right\}\right.$ \\
\hline$R_{58}=R\left(u_{4}, u_{5}\right)$ & $V\left(\operatorname{MOG}(7)-\left\{u_{1}, v_{1}, v_{2}\right\}\right.$ \\
\hline$R_{59}=R\left(u_{4}, u_{6}\right)$ & $V\left(\operatorname{MOG}(7)-\left\{u_{5}, v_{9}, v_{10}\right\}\right.$ \\
\hline$R_{60}=R\left(u_{4}, u_{7}\right)$ & $V(\operatorname{MOG}(7)-$ \\
\hline
\end{tabular}

TABle 6: Continued.

\begin{tabular}{|c|c|}
\hline Resolving sets $(n=7)$ & Elements \\
\hline$R_{61}=R\left(u_{4}, v_{1}\right)$ & $V\left(\operatorname{MOG}(7)-\left\{u_{2}, u_{7}, v_{4}, v_{12}\right\}\right.$ \\
\hline$R_{62}=R\left(u_{4}, v_{2}\right)$ & $V\left(\operatorname{MOG}(7)-\left\{u_{2}, u_{7}\right\}\right.$ \\
\hline$R_{63}=R\left(u_{4}, v_{3}\right)$ & $V\left(\operatorname{MOG}(7)-\left\{u_{7}, v_{5}, v_{13}\right\}\right.$ \\
\hline$R_{64}=R\left(u_{4}, v_{4}\right)$ & $V\left(\operatorname{MOG}(7)-\left\{u_{7}, u_{6}, v_{13}, v_{14}\right\}\right.$ \\
\hline$R_{65}=R\left(u_{4}, v_{5}\right)$ & $V\left(\operatorname{MOG}(7)-\left\{u_{2}, u_{3}, v_{1}\right\}\right.$ \\
\hline$R_{66}=R\left(u_{4}, v_{6}\right)$ & $V\left(\operatorname{MOG}(7)-\left\{u_{2}, u_{3}, u_{7}, v_{1}, v_{2}, v_{3}, v_{7}\right\}\right.$ \\
\hline$R_{67}=R\left(u_{4}, v_{7}\right)$ & $V\left(\operatorname{MOG}(7)-\left\{v_{4}, v_{5}, v_{8}, v_{9}\right\}\right.$ \\
\hline$R_{68}=R\left(u_{4}, v_{8}\right)$ & $V\left(\operatorname{MOG}(7)-\left\{v_{6}, v_{7}, v_{10}, v_{11}\right\}\right.$ \\
\hline$R_{69}=R\left(u_{4}, v_{9}\right)$ & $V\left(\operatorname{MOG}(7)-\left\{u_{5}, u_{6}, v_{7}, v_{8}, v_{12}, v_{13}\right\}\right.$ \\
\hline$R_{70}=R\left(u_{4}, v_{10}\right)$ & $V\left(\operatorname{MOG}(7)-\left\{u_{5}, u_{6}, u_{7}, v_{14}\right\}\right.$ \\
\hline$R_{71}=R\left(u_{4}, v_{11}\right)$ & $V\left(\operatorname{MOG}(7)-\left\{u_{1}, v_{1}, v_{2}, v_{9}\right\}\right.$ \\
\hline$R_{72}=R\left(u_{4}, v_{12}\right)$ & $V\left(\operatorname{MOG}(7)-\left\{u_{1}, v_{1}, v_{2}, v_{10}\right\}\right.$ \\
\hline$R_{73}=R\left(u_{4}, v_{13}\right)$ & $V\left(\operatorname{MOG}(7)-\left\{u_{6}, v_{11}\right\}\right.$ \\
\hline$R_{74}=R\left(u_{4}, v_{14}\right)$ & $V\left(\operatorname{MOG}(7)-\left\{u_{6}, v_{3}, v_{11}\right\}\right.$ \\
\hline$R_{75}=R\left(u_{5}, u_{6}\right)$ & $V\left(\operatorname{MOG}(7)-\left\{u_{2}, v_{3}, v_{4}\right\}\right.$ \\
\hline$R_{76}=R\left(u_{5}, u_{7}\right)$ & $V\left(\operatorname{MOG}(7)-\left\{u_{6}, v_{11}, v_{12}\right\}\right.$ \\
\hline$R_{77}=R\left(u_{5}, v_{1}\right)$ & $V\left(\operatorname{MOG}(7)-\left\{u_{7}\right\}\right.$ \\
\hline$R_{78}=R\left(u_{5}, v_{2}\right)$ & $V\left(\operatorname{MOG}(7)-\left\{u_{7}, u_{5}, v_{13}\right\}\right.$ \\
\hline$R_{79}=R\left(u_{5}, v_{3}\right)$ & $V\left(\operatorname{MOG}(7)-\left\{u_{3}, v_{6}, v_{14}\right\}\right.$ \\
\hline$R_{80}=R\left(u_{5}, v_{4}\right)$ & $V\left(\operatorname{MOG}(7)-\left\{u_{3}\right\}\right.$ \\
\hline$R_{81}=R\left(u_{5}, v_{5}\right)$ & $V\left(\operatorname{MOG}(7)-\left\{u_{1}, v_{1}, v_{7}\right\}\right.$ \\
\hline$R_{82}=R\left(u_{5}, v_{6}\right)$ & $V\left(\operatorname{MOG}(7)-\left\{u_{1}, v_{1}, v_{2}, v_{8}\right\}\right.$ \\
\hline$R_{83}=R\left(u_{5}, v_{7}\right)$ & $V\left(\operatorname{MOG}(7)-\left\{u_{2}, u_{3}, u_{4}, v_{3}\right\}\right.$ \\
\hline$R_{84}=R\left(u_{5}, v_{8}\right)$ & $V\left(\operatorname{MOG}(7)-\left\{u_{2}, u_{3}, u_{4}, v_{3}, v_{4}, v_{5}, v_{9}\right\}\right.$ \\
\hline$R_{85}=R\left(u_{5}, v_{9}\right)$ & $V\left(\operatorname{MOG}(7)-\left\{v_{6}, v_{7}, v_{10}, v_{11}\right\}\right.$ \\
\hline$R_{86}=R\left(u_{5}, v_{10}\right)$ & $V\left(\operatorname{MOG}(7)-\left\{v_{8}, v_{9}, v_{12}, v_{13}\right\}\right.$ \\
\hline$R_{87}=R\left(u_{5}, v_{11}\right)$ & $V\left(\operatorname{MOG}(7)-\left\{u_{1}, u_{6}, u_{7}, v_{1} v_{2}, v_{10}, v_{14}\right\}\right.$ \\
\hline$R_{88}=R\left(u_{5}, v_{12}\right)$ & $V\left(\operatorname{MOG}(7)-\left\{u_{1}, u_{6}, u_{7}, v_{2}\right\}\right.$ \\
\hline$R_{89}=R\left(u_{5}, v_{13}\right)$ & $V\left(\operatorname{MOG}(7)-\left\{u_{2}, v_{3}, v_{4}, v_{11}\right\}\right.$ \\
\hline$R_{90}=R\left(u_{5}, v_{14}\right)$ & $V\left(\operatorname{MOG}(7)-\left\{u_{2}, v_{4}, v_{12}\right\}\right.$ \\
\hline$R_{91}=R\left(u_{6}, u_{7}\right)$ & $V\left(\operatorname{MOG}(7)-\left\{u_{3}, v_{5}, v_{6}\right\}\right.$ \\
\hline$R_{92}=R\left(u_{6}, v_{1}\right)$ & $V\left(\operatorname{MOG}(7)-\left\{u_{3}, v_{5}, v_{6}, v_{13}\right\}\right.$ \\
\hline$R_{93}=R\left(u_{6}, v_{2}\right)$ & $V\left(\operatorname{MOG}(7)-\left\{u_{1}, v_{6}\right\}\right.$ \\
\hline$R_{94}=R\left(u_{6}, v_{3}\right)$ & $V\left(\operatorname{MOG}(7)-\left\{u_{1}\right\}\right.$ \\
\hline$R_{95}=R\left(u_{6}, v_{4}\right)$ & $V\left(\operatorname{MOG}(7)-\left\{u_{1}, v_{1}\right\}\right.$ \\
\hline$R_{96}=R\left(u_{6}, v_{5}\right)$ & $V\left(\operatorname{MOG}(7)-\left\{u_{1}, u_{4}, v_{2}, v_{8}\right\}\right.$ \\
\hline$R_{97}=R\left(u_{6}, v_{6}\right)$ & $V\left(\operatorname{MOG}(7)-\left\{u_{1}, u_{4}\right\}\right.$ \\
\hline$R_{98}=R\left(u_{6}, v_{7}\right)$ & $V\left(\operatorname{MOG}(7)-\left\{u_{2}, v_{3}, v_{9}\right\}\right.$ \\
\hline$R_{99}=R\left(u_{6}, v_{8}\right)$ & $V\left(\operatorname{MOG}(7)-\left\{u_{2}, v_{3}, v_{4}, v_{10}\right\}\right.$ \\
\hline$R_{100}=R\left(u_{6}, v_{9}\right)$ & $V\left(\operatorname{MOG}(7)-\left\{u_{3}, u_{4}, u_{5}, v_{5}\right\}\right.$ \\
\hline$R_{101}=R\left(u_{6}, v_{10}\right)$ & $V\left(\operatorname{MOG}(7)-\left\{u_{3}, u_{4}, u_{5}, v_{5}, v_{6}, v_{7}\right\}\right.$ \\
\hline$R_{102}=R\left(u_{6}, v_{11}\right)$ & $V\left(\operatorname{MOG}(7)-\left\{v_{8}, v_{9}, v_{12}, v_{13}\right\}\right.$ \\
\hline$R_{103}=R\left(u_{6}, v_{12}\right)$ & $V\left(\operatorname{MOG}(7)-\left\{v_{1}, v_{10}, v_{11}, v_{14}\right\}\right.$ \\
\hline$R_{104}=R\left(u_{6}, v_{13}\right)$ & $V\left(\operatorname{MOG}(7)-\left\{u_{1}, u_{2}, u_{7}, v_{2}, v_{3}, v_{4}\right\}\right.$ \\
\hline$R_{105}=R\left(u_{6}, v_{14}\right)$ & $V\left(\operatorname{MOG}(7)-\left\{u_{1}, u_{2}, u_{7}, v_{4}\right\}\right.$ \\
\hline$R_{106}=R\left(u_{7}, v_{1}\right)$ & $V\left(\operatorname{MOG}(7)-\left\{u_{1}, u_{2}, u_{3}, v_{4}, v_{5}, v_{6}, v_{14}\right\}\right.$ \\
\hline$R_{107}=R\left(u_{7}, v_{2}\right)$ & $V\left(\operatorname{MOG}(7)-\left\{u_{1}, u_{2}, u_{3}, v_{6}\right\}\right.$ \\
\hline$R_{108}=R\left(u_{7}, v_{3}\right)$ & $V\left(\operatorname{MOG}(7)-\left\{u_{4}, v_{1}, v_{7}, v_{8}\right\}\right.$ \\
\hline$R_{109}=R\left(u_{7}, v_{4}\right)$ & $V\left(\operatorname{MOG}(7)-\left\{u_{4}, v_{2}, v_{8}\right\}\right.$ \\
\hline$R_{110}=R\left(u_{7}, v_{5}\right)$ & $V\left(\operatorname{MOG}(7)-\left\{u_{2}\right\}\right.$ \\
\hline$R_{111}=R\left(u_{7}, v_{6}\right)$ & $V\left(\operatorname{MOG}(7)-\left\{u_{2}, v_{3}, v_{9}\right\}\right.$ \\
\hline$R_{112}=R\left(u_{7}, v_{7}\right)$ & $V\left(\operatorname{MOG}(7)-\left\{u_{5}, v_{4}, v_{10}\right\}\right.$ \\
\hline$R_{113}=R\left(u_{7}, v_{8}\right)$ & $V\left(\operatorname{MOG}(7)-\left\{u_{5}, v_{4}\right\}\right.$ \\
\hline$R_{114}=R\left(u_{7}, v_{9}\right)$ & $V\left(\operatorname{MOG}(7)-\left\{u_{3}, v_{5}, v_{11}\right\}\right.$ \\
\hline$R_{115}=R\left(u_{7}, v_{10}\right)$ & $V\left(\operatorname{MOG}(7)-\left\{u_{3}, v_{5}, v_{6}, v_{12}\right\}\right.$ \\
\hline$R_{116}=R\left(u_{7}, v_{11}\right)$ & $V\left(\operatorname{MOG}(7)-\left\{u_{4}, u_{5}, u_{6}, v_{7}\right\}\right.$ \\
\hline$R_{117}=R\left(u_{7}, v_{12}\right)$ & $V\left(\operatorname{MOG}(7)-\left\{u_{4}, u_{5}, u_{6}, v_{7}, v_{8}, v_{9}, v_{13}\right\}\right.$ \\
\hline$R_{118}=R\left(u_{7}, v_{13}\right)$ & $V\left(\operatorname{MOG}(7)-\left\{v_{1}, v_{10}, v_{11}, v_{14}\right\}\right.$ \\
\hline$R_{119}=R\left(u_{7}, v_{14}\right)$ & $V\left(\operatorname{MOG}(7)-\left\{v_{2}, v_{3}, v_{12}, v_{13}\right\}\right.$ \\
\hline$R_{120}=R\left(v_{1}, v_{3}\right)$ & $V\left(\operatorname{MOG}(7)-\left\{u_{5}, v_{2}, v_{9}, v_{10}\right\}\right.$ \\
\hline
\end{tabular}


Table 6: Continued.

\begin{tabular}{|c|c|}
\hline Resolving sets $(n=7)$ & Elements \\
\hline$R_{121}=R\left(v_{1}, v_{4}\right)$ & $V\left(\operatorname{MOG}(7)-\left\{u_{5}, v_{9}, v_{10}\right\}\right.$ \\
\hline$R_{122}=R\left(v_{1}, v_{5}\right)$ & $V\left(\operatorname{MOG}(7)-\left\{u_{2}, v_{3}\right\}\right.$ \\
\hline$R_{123}=R\left(v_{1}, v_{6}\right)$ & $V\left(\operatorname{MOG}(7)-\left\{u_{2}\right\}\right.$ \\
\hline$R_{124}=R\left(v_{1}, v_{7}\right)$ & $V\left(\operatorname{MOG}(7)-\left\{u_{6}, v_{4}, v_{11}\right\}\right.$ \\
\hline$R_{125}=R\left(v_{1}, v_{8}\right)$ & $V\left(\operatorname{MOG}(7)-\left\{u_{6}\right\}\right.$ \\
\hline$R_{126}=R\left(v_{1}, v_{9}\right)$ & $V\left(\operatorname{MOG}(7)-\left\{u_{3}, v_{5}, v_{12}\right\}\right.$ \\
\hline$R_{127}=R\left(v_{1}, v_{10}\right)$ & $V\left(\operatorname{MOG}(7)-\left\{u_{3}, v_{5}, v_{6}\right\}\right.$ \\
\hline$R_{128}=R\left(v_{1}, v_{11}\right)$ & $V\left(\operatorname{MOG}(7)-\left\{u_{7}, v_{13}\right\}\right.$ \\
\hline$R_{129}=R\left(v_{1}, v_{12}\right)$ & $V\left(\operatorname{MOG}(7)-\left\{u_{7}\right\}\right.$ \\
\hline$R_{130}=R\left(v_{1}, v_{13}\right)$ & $V\left(\operatorname{MOG}(7)-\left\{u_{4}, v_{7}, v_{8}, v_{14}\right\}\right.$ \\
\hline$R_{131}=R\left(v_{1}, v_{14}\right)$ & $V\left(\operatorname{MOG}(7)-\left\{u_{4}, v_{7}, v_{8}\right\}\right.$ \\
\hline$R_{132}=R\left(v_{2}, v_{3}\right)$ & $V\left(\operatorname{MOG}(7)-\left\{u_{5}, v_{9}, v_{10}\right\}\right.$ \\
\hline$R_{133}=R\left(v_{2}, v_{4}\right)$ & $V\left(\operatorname{MOG}(7)-\left\{u_{5}, v_{3}, v_{9}, v_{10}\right\}\right.$ \\
\hline$R_{134}=R\left(v_{2}, v_{5}\right)$ & $V\left(\operatorname{MOG}(7)-\left\{u_{2}\right\}\right.$ \\
\hline$R_{135}=R\left(v_{2}, v_{6}\right)$ & $V\left(\operatorname{MOG}(7)-\left\{u_{2}, v_{4}\right\}\right.$ \\
\hline$R_{136}=R\left(v_{2}, v_{7}\right)$ & $V\left(\operatorname{MOG}(7)-\left\{u_{6}, u_{11}, v_{12}\right\}\right.$ \\
\hline$R_{137}=R\left(v_{2}, v_{8}\right)$ & $V\left(\operatorname{MOG}(7)-\left\{u_{6}, v_{5}, v_{12}\right\}\right.$ \\
\hline$R_{138}=R\left(v_{2}, v_{9}\right)$ & $V\left(\operatorname{MOG}(7)-\left\{u_{3}\right\}\right.$ \\
\hline$R_{139}=R\left(v_{2}, v_{10}\right)$ & $V\left(\operatorname{MOG}(7)-\left\{u_{3}, v_{6}, v_{13}\right\}\right.$ \\
\hline$R_{140}=R\left(v_{2}, v_{11}\right)$ & $V\left(\operatorname{MOG}(7)-\left\{u_{7}\right\}\right.$ \\
\hline$R_{141}=R\left(v_{2}, v_{12}\right)$ & $V\left(\operatorname{MOG}(7)-\left\{u_{7}, v_{14}\right\}\right.$ \\
\hline$R_{142}=R\left(v_{2}, v_{13}\right)$ & $V\left(\operatorname{MOG}(7)-\left\{u_{4}, v_{7}, v_{8}\right\}\right.$ \\
\hline$R_{143}=R\left(v_{2}, v_{14}\right)$ & $V\left(\operatorname{MOG}(7)-\left\{u_{4}, v_{1}, v_{7}, v_{8}\right\}\right.$ \\
\hline$R_{144}=R\left(v_{3}, v_{5}\right)$ & $V\left(\operatorname{MOG}(7)-\left\{u_{6}, v_{4}, v_{11}, v_{12}\right\}\right.$ \\
\hline$R_{145}=R\left(v_{3}, v_{6}\right)$ & $V\left(\operatorname{MOG}(7)-\left\{u_{6}, v_{11}, v_{12}\right\}\right.$ \\
\hline$R_{146}=R\left(v_{3}, v_{7}\right)$ & $V\left(\operatorname{MOG}(7)-\left\{u_{3}, v_{5}\right\}\right.$ \\
\hline$R_{147}=R\left(v_{3}, v_{8}\right)$ & $V\left(\operatorname{MOG}(7)-\left\{u_{3}\right\}\right.$ \\
\hline$R_{148}=R\left(v_{3}, v_{9}\right)$ & $V\left(\operatorname{MOG}(7)-\left\{u_{7}, v_{6}, v_{13}\right\}\right.$ \\
\hline$R_{149}=R\left(v_{3}, v_{10}\right)$ & $V\left(\operatorname{MOG}(7)-\left\{u_{7}\right\}\right.$ \\
\hline$R_{150}=R\left(v_{3}, v_{11}\right)$ & $V\left(\operatorname{MOG}(7)-\left\{u_{4}, v_{7}, v_{14}\right\}\right.$ \\
\hline$R_{151}=R\left(v_{3}, v_{12}\right)$ & $V\left(\operatorname{MOG}(7)-\left\{u_{4}, v_{7}, v_{8}\right\}\right.$ \\
\hline$R_{152}=R\left(v_{3}, v_{13}\right)$ & $V\left(\operatorname{MOG}(7)-\left\{u_{1}, v_{1}\right\}\right.$ \\
\hline$R_{153}=R\left(v_{3}, v_{14}\right)$ & $V\left(\operatorname{MOG}(7)-\left\{u_{1}\right\}\right.$ \\
\hline$R_{154}=R\left(v_{4}, v_{5}\right)$ & $V\left(\operatorname{MOG}(7)-\left\{u_{6}, v_{11}, v_{12}\right\}\right.$ \\
\hline$R_{155}=R\left(v_{4}, v_{6}\right)$ & $V\left(\operatorname{MOG}(7)-\left\{u_{6}, v_{5}, v_{11}, v_{12}\right\}\right.$ \\
\hline$R_{156}=R\left(v_{4}, v_{7}\right)$ & $V\left(\operatorname{MOG}(7)-\left\{u_{3}\right\}\right.$ \\
\hline$R_{157}=R\left(v_{4}, v_{8}\right)$ & $V\left(\operatorname{MOG}(7)-\left\{u_{3}, v_{6}\right\}\right.$ \\
\hline$R_{158}=R\left(v_{4}, v_{9}\right)$ & $V\left(\operatorname{MOG}(7)-\left\{u_{7}, v_{13}, v_{14}\right\}\right.$ \\
\hline$R_{159}=R\left(v_{4}, v_{10}\right)$ & $V\left(\operatorname{MOG}(7)-\left\{u_{7}, v_{7}, v_{14}\right\}\right.$ \\
\hline$R_{160}=R\left(v_{4}, v_{11}\right)$ & $V\left(\operatorname{MOG}(7)-\left\{u_{4}\right\}\right.$ \\
\hline$R_{161}=R\left(v_{4}, v_{12}\right)$ & $V\left(\operatorname{MOG}(7)-\left\{u_{4}, v_{1}, v_{8}\right\}\right.$ \\
\hline$R_{162}=R\left(v_{4}, v_{13}\right)$ & $V\left(\operatorname{MOG}(7)-\left\{u_{1}\right\}\right.$ \\
\hline$R_{163}=R\left(v_{4}, v_{14}\right)$ & $V\left(\operatorname{MOG}(7)-\left\{u_{1}, v_{2}\right\}\right.$ \\
\hline$R_{164}=R\left(v_{5}, v_{7}\right)$ & $V\left(\operatorname{MOG}(7)-\left\{u_{7}, v_{6}\right\}\right.$ \\
\hline$R_{165}=R\left(v_{5}, v_{8}\right)$ & $V\left(\operatorname{MOG}(7)-\left\{u_{7}, u_{12}, v_{14}\right\}\right.$ \\
\hline$R_{166}=R\left(v_{5}, v_{9}\right)$ & $V\left(\operatorname{MOG}(7)-\left\{u_{4}, v_{7}\right\}\right.$ \\
\hline$R_{167}=R\left(v_{5}, v_{10}\right)$ & $V\left(\operatorname{MOG}(7)-\left\{u_{4}\right\}\right.$ \\
\hline$R_{168}=R\left(v_{5}, v_{11}\right)$ & $V\left(\operatorname{MOG}(7)-\left\{u_{1}, v_{1}, v_{8}\right\}\right.$ \\
\hline$R_{169}=R\left(v_{5}, v_{12}\right)$ & $V\left(\operatorname{MOG}(7)-\left\{u_{1}\right\}\right.$ \\
\hline$R_{170}=R\left(v_{5}, v_{13}\right)$ & $V\left(\operatorname{MOG}(7)-\left\{u_{5}, v_{2}, v_{9}\right\}\right.$ \\
\hline$R_{171}=R\left(v_{5}, v_{14}\right)$ & $V\left(\operatorname{MOG}(7)-\left\{u_{5}, v_{9}, v_{10}\right\}\right.$ \\
\hline$R_{172}=R\left(v_{6}, v_{7}\right)$ & $V\left(\operatorname{MOG}(7)-\left\{u_{7}, v_{13}, v_{14}\right\}\right.$ \\
\hline$R_{173}=R\left(v_{6}, v_{8}\right)$ & $V\left(\operatorname{MOG}(7)-\left\{u_{7}, v_{7}, v_{13}, v_{14}\right\}\right.$ \\
\hline$R_{174}=R\left(v_{6}, v_{9}\right)$ & $V\left(\operatorname{MOG}(7)-\left\{u_{4}\right\}\right.$ \\
\hline$R_{175}=R\left(v_{6}, v_{10}\right)$ & $V\left(\operatorname{MOG}(7)-\left\{u_{4}, v_{8},\right\}\right.$ \\
\hline$R_{176}=R\left(v_{6}, v_{11}\right)$ & $V\left(\operatorname{MOG}(7)-\left\{u_{1}, v_{1}, v_{2}\right\}\right.$ \\
\hline$R_{177}=R\left(v_{6}, v_{12}\right)$ & $V\left(\operatorname{MOG}(7)-\left\{u_{1}, v_{2}, v_{9}\right\}\right.$ \\
\hline$R_{178}=R\left(v_{6}, v_{13}\right)$ & $V\left(\operatorname{MOG}(7)-\left\{u_{5}\right\}\right.$ \\
\hline$R_{179}=R\left(v_{6}, v_{14}\right)$ & $V\left(\operatorname{MOG}(7)-\left\{u_{5}, v_{3}, v_{10}\right\}\right.$ \\
\hline$R_{180}=R\left(v_{7}, v_{9}\right)$ & $V\left(\operatorname{MOG}(7)-\left\{u_{1}, v_{1}, v_{2}, v_{8}\right\}\right.$ \\
\hline
\end{tabular}

TABle 6: Continued.

\begin{tabular}{|c|c|}
\hline Resolving sets $(n=7)$ & Elements \\
\hline$R_{181}=R\left(v_{7}, v_{10}\right)$ & $V\left(\operatorname{MOG}(7)-\left\{u_{1}, v_{1}, v_{2}\right\}\right.$ \\
\hline$R_{182}=R\left(v_{7}, v_{11}\right)$ & $V\left(\operatorname{MOG}(7)-\left\{u_{5}, v_{9}\right\}\right.$ \\
\hline$R_{183}=R\left(v_{7}, v_{12}\right)$ & $V\left(\operatorname{MOG}(7)-\left\{u_{5}\right\}\right.$ \\
\hline$R_{184}=R\left(v_{7}, v_{13}\right)$ & $V\left(\operatorname{MOG}(7)-\left\{u_{3}, v_{3}, v_{10}\right\}\right.$ \\
\hline$R_{185}=R\left(v_{7}, v_{14}\right)$ & $V\left(\operatorname{MOG}(7)-\left\{u_{2}\right\}\right.$ \\
\hline$R_{186}=R\left(v_{8}, v_{9}\right)$ & $V\left(\operatorname{MOG}(7)-\left\{u_{1}, v_{1}, v_{2}\right\}\right.$ \\
\hline$R_{187}=R\left(v_{8}, v_{10}\right)$ & $V\left(\operatorname{MOG}(7)-\left\{u_{1}, v_{1}, v_{2}, v_{9}\right\}\right.$ \\
\hline$R_{188}=R\left(v_{8}, v_{11}\right)$ & $V\left(\operatorname{MOG}(7)-\left\{u_{5}\right\}\right.$ \\
\hline$R_{189}=R\left(v_{8}, v_{12}\right)$ & $V\left(\operatorname{MOG}(7)-\left\{u_{5}, v_{10}\right\}\right.$ \\
\hline$R_{190}=R\left(v_{8}, v_{13}\right)$ & $V\left(\operatorname{MOG}(7)-\left\{u_{2}, v_{3}, v_{4}\right\}\right.$ \\
\hline$R_{191}=R\left(v_{8}, v_{14}\right)$ & $V\left(\operatorname{MOG}(7)-\left\{u_{2}, v_{4}, v_{11}\right\}\right.$ \\
\hline$R_{192}=R\left(v_{9}, v_{11}\right)$ & $V\left(\operatorname{MOG}(7)-\left\{u_{2}, v_{3}, v_{4}, v_{10}\right\}\right.$ \\
\hline$R_{193}=R\left(v_{9}, v_{12}\right)$ & $V\left(\operatorname{MOG}(7)-\left\{u_{2}, v_{3}, v_{4}\right\}\right.$ \\
\hline$R_{194}=R\left(v_{9}, v_{13}\right)$ & $V\left(\operatorname{MOG}(7)-\left\{u_{6}, v_{11}\right\}\right.$ \\
\hline$R_{195}=R\left(v_{9}, v_{14}\right)$ & $V\left(\operatorname{MOG}(7)-\left\{u_{6}\right\}\right.$ \\
\hline$R_{196}=R\left(v_{10}, v_{11}\right)$ & $V\left(\operatorname{MOG}(7)-\left\{u_{2}, v_{3}, v_{4}\right\}\right.$ \\
\hline$R_{197}=R\left(v_{10}, v_{12}\right)$ & $V\left(\operatorname{MOG}(7)-\left\{u_{2}, v_{3}, v_{4}, v_{11}\right\}\right.$ \\
\hline$R_{198}=R\left(v_{10}, v_{13}\right)$ & $V\left(\operatorname{MOG}(7)-\left\{u_{6}\right\}\right.$ \\
\hline$R_{199}=R\left(v_{10}, v_{14}\right)$ & $V\left(\operatorname{MOG}(7)-\left\{u_{6}, v_{12}\right\}\right.$ \\
\hline$R_{200}=R\left(v_{11}, v_{13}\right)$ & $V\left(\operatorname{MOG}(7)-\left\{u_{3}, v_{5}, v_{6}, v_{12}\right\}\right.$ \\
\hline$R_{201}=R\left(v_{11}, v_{14}\right)$ & $V\left(\operatorname{MOG}(7)-\left\{u_{3}, v_{5}, v_{6}\right\}\right.$ \\
\hline$R_{202}=R\left(v_{12}, v_{13}\right)$ & $V\left(\operatorname{MOG}(7)-\left\{u_{3}, v_{5}, v_{6}\right\}\right.$ \\
\hline$R_{203}=R\left(v_{12}, v_{14}\right)$ & $V\left(\operatorname{MOG}(7)-\left\{u_{3}, v_{5}, v_{6}, v_{13}\right\}\right.$ \\
\hline
\end{tabular}

TABLE 7: FMD of metal organic graphs.

\begin{tabular}{lc}
\hline $\operatorname{MOG}(n)$ and $n \equiv 1(\bmod 2)$ & Upper bounds of FMD \\
\hline $\operatorname{MOG}(3)$ & $3 / 2$ \\
$\operatorname{MOG}(5)$ & $5 / 4$ \\
$\operatorname{MOG}(7)$ & $7 / 4$ \\
$\operatorname{MOG}(n)$ if $n \geq 9$ & $n / 4$
\end{tabular}

\section{Conclusion}

In this section, we conclude the obtained results as follows:

(i) The FMD of $\operatorname{MOG}(n)$ for $n \equiv 1(\bmod 2)$ is obtained as given in Table 7 .

(ii) We note that as we increase $n$ in $\operatorname{MOG}(n)$ for $n \equiv 1(\bmod 2)$, the FMD also increases.

(iii) This is one of the important graphs that has same FMD and LFMD having unique resolving and local resolving neighbourhood sets.

(iv) The problem is still open to characterize the graphs with same FMD and LFMD.

\section{Data Availability}

The data used to support the finding of this study are included within the article. Additional data can be obtained from the corresponding author upon request.

\section{Disclosure}

There is no funding source. 


\section{Conflicts of Interest}

The authors declare that there are no conflicts of interest.

\section{References}

[1] F. Harary and R. A. Melter, "The metric dimension of a graph,” Ars Combinatorica, vol. 2, pp. 191-195, 1976.

[2] G. Chartrand, L. Eroh, M. A. Johnson, and O. R. Oellermann, "Resolvability in graphs and the metric dimension of a graph," Discrete Applied Mathematics, vol. 105, no. 1-3, pp. 99-113, 2000.

[3] J. Currie and O. R. Oellermann, "The metric dimension and metric independence of a graph," Journal of Combinatorial Mathematics and Combinatorial Computing, vol. 39, pp. 157-167, 2001.

[4] Z. Beerliova, F. Eberhard, T. Erlebach et al., "Network discovery and verification," IEEE Journal on Selected Areas in Communications, vol. 24, no. 12, pp. 2168-2181, 2006.

[5] S. Khuller, B. Raghavachari, and A. Rosenfeld, Localization in graphs, CCR-93-07462 DACA76-92-C-0009, 1994.

[6] Y.-M. Chu, M. F. Nadeem, M. Azeem, and M. K. Siddiqui, "On sharp bounds on partition dimension of convex polytopes," IEEE Access, vol. 8, pp. 224781-224790, 2020.

[7] J. Liu and M. F. Nadeem, "Bounds on the partition dimension of convex polytopes," Combinatorial Chemistry and High Throughput Screening, vol. 23, 2020.

[8] M. F. Nadeem, M. Azeem, and A. Khalil, "The locating number of hexagonal Möbius ladder network," Journal of Applied Mathematics and Computing, 2020.

[9] Z. S. Mufti, M. F. Nadeem, A. Ahmad, and Z. Ahmad, "Computation of edge metric dimension of barycentric subdivision of cayley graphs," Italian Journal of Pure and Applied Mathematics, vol. 44, pp. 714-722, 2020.

[10] M. Fehr, S. Gosselin, and O. R. Oellermann, "The metric dimension of Cayley digraphs," Discrete Mathematics, vol. 306, no. 1, pp. 31-41, 2006.

[11] S. Arumugam and V. Mathew, "The fractional metric dimension of graphs," Discrete Mathematics, vol. 312, no. 9, pp. 1584-1590, 2012.

[12] M. Raza, M. Javaid, and N. Saleem, Fractional metric dimension of metal organic frameworks, main group metal chemistry in press, 2021.

[13] S. Arumugam and V. Mathew, "The fractional metric dimension of graphs," Discrete Mathematics, vol. 5, pp. 1-8, 2013.

[14] M. Feng and K. Wang, "On the metric dimension and fractional metric dimension for hierarchical product of graphs," Applicable Analysis and Discrete Mathematics, vol. 7, no. 2, pp. 302-313, 2013.

[15] M. Feng and Q. Kong, "On the fractional metric dimension of corona product graphs and lexicographic product graphs," Ars Combinatorica, vol. 138, pp. 249-260, 2018.

[16] S. W. Saputro, A. S. Fenovcikova, M. Baca, and M. Lascsakova, "On fractional metric dimension of comb product graphs," Statistics, Optimization and Information Computing, vol. 6, pp. 150-158, 2018.

[17] E. Yi, "The fractional metric dimension of permutation graphs," Acta Mathematica Sinica, English Series, vol. 31, no. 3, pp. 367-382, 2015.

[18] J. Liu, A. Kashif, T. Rashid, and M. Javaid, "Fractional metric dimension of generalized Jahangir graph," Mathematics, vol. 7, pp. 1-10, 2019.
[19] S. Aisyah, U. M. Imam, and L. Susilowati, "Local fractional metric dimension of cornea product of graphs," IOP Conference Series: Earth and Environmental Science, vol. 243, 2019.

[20] J.-B. Liu, M. K. Aslam, and M. Javaid, "Local fractional metric dimensions of rotationally symmetric and planar networks," IEEE Access, vol. 8, pp. 82404-82420, 2020.

[21] M. Javaid, M. Raza, P. Kumam, and J.-B. Liu, "Sharp bounds of local fractional metric dimensions of connected networks," IEEE Access, vol. 8, pp. 172329-172342, 2020.

[22] J. Liu, J. Y. Ruseow, W. Scotskinner, and Z. U. Wang, "Metal organic frameworks: structures and functional applications," Materials Today, vol. 27, pp. 43-68, 2019.

[23] C. Pettinari, F. Marchetti, N. Mosca, G. Tosi, and A. Drozdov, "Application of metal - organic frameworks," Polymer International, vol. 66, no. 6, pp. 731-744, 2017.

[24] J. Liu, L. Chen, H. Cui, J. Zhang, L. Zhang, and C.-Y. Su, "Applications of metal-organic frameworks in heterogeneous supramolecular catalysis," Chemical Society Reviews, vol. 43, no. 16, pp. 6011-6061, 2014.

[25] Z. Hasan and S. H. Jhung, "Removal of hazardous organics from water using metal-organic frameworks (MOFs): plausible mechanisms for selective adsorptions," Journal of Hazardous Materials, vol. 283, pp. 329-339, 2015. 